\title{
Random phaseless sampling for causal signals in shift-invariant spaces: a zero distribution perspective
}

\author{
Youfa Li, Wenchang Sun
}

\begin{abstract}
We proved that the phaseless sampling (PLS) in the linear-phase modulated shift-invariant space (SIS) $V\left(e^{\mathbf{i} \alpha \cdot} \varphi\right), \alpha \neq 0$, is impossible even though the real-valued function $\varphi$ enjoys the full spark property (so does $e^{i \alpha \cdot} \varphi$ ). Stated another way, the PLS in the complex-generated SISs is essentially different from that in the real-generated ones. Motivated by this, we first establish the condition on the complex-valued generator $\phi$ such that the PLS of nonseparable causal (NC) signals in $V(\phi)$ can be achieved by random sampling. The condition is established from the generalized Haar condition (GHC) perspective. Based on the proposed reconstruction approach, it is proved that if the GHC holds then with probability 1 , the random sampling density (SD) $=3$ is sufficient for the PLS of NC signals in the complex-generated SISs. For the realvalued case we also prove that, if the GHC holds then with probability 1 , the random $S D=2$ is sufficient for the PLS of real-valued NC signals in the real-generated SISs. For the local reconstruction of highly oscillatory signals such as chirps, a great number of deterministic samples are required. Compared with deterministic sampling, the proposed random approach enjoys not only the greater sampling flexibility but the much smaller number of samples. To verify our results, numerical simulations were conducted to reconstruct highly oscillatory NC signals in the chirp-modulated SISs.
\end{abstract}

Index Terms-Random phaseless sampling, complex (real)generated shift-invariant space, generalized Haar condition, sampling density, highly oscillatory signals.

\section{INTRODUCTION}

Phase retrieval (PR) is a nonlinear problem that seeks to reconstruct a signal $f$, up to a unimodular scalar, from

Youfa Li. College of Mathematics and Information Science, Guangxi University, Naning 530004, China. Email: youfalee@ hotmail.com

Wenchang Sun. School of Mathematical Sciences and LPMC, Nankai University, Tianjin 300071, China. Email:sunwch@nankai.edu.cn

Youfa $\mathrm{Li}$ is partially supported by Natural Science Foundation of China (Nos: 61961003, 61561006, 11501132), Natural Science Foundation of Guangxi (No: 2019GXNSFAA185035) and the talent project of Education Department of Guangxi Government for Young-MiddleAged backbone teachers. Wenchang Sun was partially supported by the National Natural Science Foundation of China (11525104, 11531013 and 11761131002) the intensities of the linear measurements (c.f.[1], [2], [3], [4], [5], [6], [7])

$$
b_{k}:=\left|\left\langle f, \mathbf{a}_{k}\right\rangle\right|, k \in \Gamma,
$$

where $\mathbf{a}_{k}$ is called the measurement vector.

As stated in Y. Shechtman et. al [7] one of the reasons for PR in optics is that, for highly oscillatory signals such as optical waves (electromagnetic fields oscillating at $10^{15}$ $\mathrm{Hz}$ and higher), measuring their phases is very difficult or even impossible for electronic measurement devices. PR has been widely investigated in engineering and mathematical problems such as coherent diffraction imaging ([7], [8], [9]), quantum tomography ([10]), and frame theory ([11], [12]). A concrete PR problem corresponds to the specific signal class $\mathcal{C}$ and measurement vectors (e.g. [13], [14], [15], [16], [17], [18], [19]). For example, Alaifari et. al [15] considered the PR of real-valued bandlimited functions by frame measurement vectors. When $f$ lies in a function class $\mathcal{C}$ and $\mathbf{a}_{k}$ is the shift of the Dirac distribution, then the corresponding $\mathrm{PR}$ is the phaseless sampling (PLS for short), modeled as

$$
\text { to reconstruct } f \text { by the samples }|f(x)|, x \in \Omega \text {, }
$$

up to a unimodular scalar. In what follows, we introduce the recent developments on PLS in shift-invariant spaces (SISs).

\section{A. Related work}

SIS has many applications in signal processing. Please refer to [19], [20], [21], [22] and the references therein for a few examples. For a generator $g: \mathbb{R} \rightarrow \mathbb{C}$, its SIS is defined as

$$
V(g):=\left\{\sum_{k \in \mathbb{Z}} c_{k} g(\cdot-k):\left\{c_{k}\right\}_{k \in \mathbb{Z}} \in \ell^{2}\right\},
$$

where $\left\{c_{k}\right\}_{k \in \mathbb{Z}} \in \ell^{2}$ means $\sum_{k \in \mathbb{Z}}\left|c_{k}\right|^{2}<\infty$. Recently, PLS in SISs received much attention (e.g.[23], [24], [25], [26], [27], [28]). Particularly, it was investigated for bandlimited signals in Thakur [25], P. Jaming, K. Kellay and R. Perez Iii [28] and C.K. Lai, F. Littmann, E. 
Weber [29]. Note that the spaces of bandlimited signals are shift-invariant and the corresponding generators (sinc function or its dilations) are infinitely supported (c.f. [30], [31]). Chen, Cheng, Sun and Wang [26] established the PLS of nonseparable (the definition of nonseparability is postponed to section I-B1) real-valued signals in the SIS from a compactly supported generator. W. Sun [24] established the PLS for nonseparable real-valued signals in SISs generated by B-splines.

Note that the generators and signals in [24], [26] are all real-valued, and the sampling is deterministic. Motivated by the results therein we will investigate the random PLS of causal signals in complex (or real)-generated SISs. Here a signal $f \in V(g)$ is said to be causal if

$$
f=\sum_{k=0}^{\infty} c_{k} g(\cdot-k), c_{0} \neq 0 .
$$

The set of causal signals in $V(g)$ is denoted by $V_{\mathrm{ca}}(g)$. Causal signals are an important class of signals (c.f. [19], [32], [33]). Particularly, the Fourier measurement-based PR of causal signals in SISs was addressed in [19]. In what follows, we introduce the motivation.

\section{B. Motivation}

1) Full spark property fails for complex-valued case: Many practical applications require processing signals in the SISs from complex-valued generators such as chirps (e.g.[21], [34]). We will investigate the PLS in complexgenerated SISs. To the best of our knowledge, there are few literatures on this topic. We are greatly motivated by Theorem 1.1, which will state that the PLS in the complexgenerated SISs is essentially different from that in the realgenerated ones.

Some denotations and definitions are necessary for Theorem 1.1. A nonzero function $f$ is traditionally denoted as $f \not \equiv 0$, and $f(x) \neq 0$ means that the point $x$ is not the zero of $f$. The conjugate of $a \in \mathbb{C}$ is denoted by $\bar{a}$. The real and imaginary parts of $a$ are denoted by $\Re(a)$ and $\Im(a)$, respectively. Any $a \neq 0$ can be denoted by $|a| e^{\mathbf{i} \theta(a)}$ where $\mathbf{i},|a|$ and $\theta(a)$ are the imaginary unit, modulus and phase, respectively. For phases $\theta(a)$ and $\theta(b)$, we say that $\theta(a)=\theta(b)$ if $\theta(a)=\theta(b)+2 k \pi$ for a certain $k \in \mathbb{Z}$. Traditionally, the phase of zero can be assigned arbitrarily.

Throughout this paper the complex and real-valued generators are denoted by $\phi$ and $\varphi$, respectively. Without loss of generality, assume that

$$
\operatorname{supp}(\phi) \subseteq(0, s), \operatorname{supp}(\varphi) \subseteq(0, s)
$$

with the integer $s \geq 2$. A function $0 \not \equiv f \in V(\phi)$ (or $V(\varphi))$ is separable if there exist $0 \not \equiv f_{1}$ and $0 \not \equiv f_{2} \in$ $V(\phi)$ (or $V(\varphi)$ ) such that $f=f_{1}+f_{2}$ and $f_{1} f_{2} \equiv 0$. Clearly, if $f$ is separable then $|f|=\left|f_{1}+e^{\mathbf{i} \alpha} f_{2}\right|$ where $\alpha \in(0,2 \pi)$, and consequently it is not distinguishable from $f_{1}+e^{\mathbf{i} \alpha} f_{2}$ by the samples of $|f|$.

For the above real-valued generator $\varphi$, if the matrix

$$
\left(\varphi\left(x_{k}+n\right)\right)_{1 \leq k \leq 2 s-1,0 \leq n \leq s-1}
$$

is full spark (c.f. [35], [36]) for any $2 s-1$ distinct points $x_{k} \in(0,1), k=1, \ldots, 2 s-1$, namely, every $s \times s$ submatrix is nonsingular, then it follows from [26] that the realvalued nonseparable signals in $V(\varphi)$ can be determined by sufficiently many samples. The B-spline generators in [24] satisfy the property. However, the following theorem implies that the property is not sufficient for achieving PLS when the generator is complex-valued.

Theorem 1.1: Let $\varphi$ be real-valued such that $\operatorname{supp}(\varphi) \subseteq$ $(0, s)$ and the matrix in (1.2) is full spark for any $2 s-1$ distinct points $x_{k} \in(0,1), k=1, \ldots, 2 s-1$. Define $\phi:=e^{\mathbf{i} \alpha \cdot} \varphi$ with $\alpha \neq 0$. Then the PLS in $V_{\text {ca }}(\phi)$ can not be achieved despite the fact that $\phi$ also satisfies the full spark property.

Proof: It is easy to check that $\phi$ inherits the full spark property of $\varphi$. It follows from the full spark property that $\{\phi(\cdot+k): k=0, \ldots, s-1\}$ is linearly independent. We first choose $\beta \in \mathbb{R}$ such that $\alpha-\beta \neq k \pi$ for any $k \in \mathbb{Z}$. Let $N \geq 2$. Define a sequence $\left\{c_{k}\right\}_{k=0}^{N}$ such that $c_{0}=1$ and $c_{1}=e^{\mathbf{i} \beta}$. It is easy to check that $\left\{c_{k}\right\}_{k=0}^{N} \neq e^{\mathbf{i} \hat{\theta}}\left\{e^{\mathbf{i} 2 \alpha k} \bar{c}_{k}\right\}_{k=0}^{N}$ for any $\widehat{\theta} \in[0,2 \pi)$. By the above linear independence, we have $\sum_{k=0}^{N} c_{k} \phi(\cdot-k) \neq$ $e^{\mathbf{i} \widehat{\theta}} \sum_{k=0}^{N} e^{\mathbf{i} 2 \alpha k} \bar{c}_{k} \phi(\cdot-k)$. However, it is easy to check that

$$
\left|\sum_{k=0}^{N} e^{\mathrm{i} 2 \alpha k} \bar{c}_{k} \phi(\cdot-k)\right|=\left|\sum_{k=0}^{N} c_{k} \phi(\cdot-k)\right| .
$$

In other words, the PLS in $V_{\mathrm{ca}}(\phi)$ can not be achieved.

Motivated by Theorem 1.1, we need to establish a condition on the complex-valued generator $\phi$ such that the PLS in $V_{\mathrm{ca}}(\phi)$ can be achieved. The condition will be established from the zero distribution (or the Lebesgue measure of zero set) perspective. Our motivation for this perspective is introduced in what follows.

2) New perspective: zero distribution-based PLS:

We first interpret the full spark property from the zero distribution perspective. For a function system $\Lambda=$ $\left\{g_{0}, \ldots, g_{L-1}\right\}$, its span space is defined as

$$
\operatorname{span}\{\Lambda\}:=\left\{\sum_{j=0}^{L-1} c_{j} g_{j}: c_{j} \in \mathbb{R}\right\} .
$$

It is easy to check that the full spark property of the matrix in (1.2) is equivalent to that the function system

$$
\Lambda_{\varphi}:=\{\varphi, \ldots, \varphi(\cdot+s-1)\}
$$


satisfies the $(s-1)$-Haar condition (HC for short) on $(0,1)$ (c.f.[37], [38], [39] for HC). Specifically, $\Lambda_{\varphi}$ is linearly independent and

$$
\sup _{0 \neq h \in \operatorname{span}\left\{\Lambda_{\varphi}\right\}} \#\left(\mathcal{Z}_{h} \cap(0,1)\right) \leq s-1,
$$

where $\mathcal{Z}_{h}$ is the zero set of $h$ and $\#\left(\mathcal{Z}_{h} \cap(0,1)\right)$ is the cardinality of $\mathcal{Z}_{h} \cap(0,1)$.

Motivated by the above $\mathrm{HC}$, from the zero distribution perspective we will establish the condition on $\phi:=\phi_{\Re}+$ $\mathbf{i} \phi_{\Im}$ such that the PLS in $V_{\text {ca }}(\phi)$ can be achieved. Inspired by Theorem 1.1, the zero distribution should not be correlated with the functions in $\operatorname{span}\{\phi, \ldots, \phi(\cdot+s-1)\}$. Instead we will require in section II that the distribution is related with the functions in $\operatorname{span}\left(\Xi_{\phi}\right)$, where

$$
\begin{aligned}
\Xi_{\phi}:= & \left\{\phi_{\Re} \phi_{\Re}(\cdot+k)+\phi_{\Im} \phi_{\Im}(\cdot+k), \phi_{\Re} \phi_{\Im}(\cdot+k)-\right. \\
& \left.\phi_{\Im} \phi_{\Re}(\cdot+k)\right\}_{k=1}^{s-1} \cup\left\{\phi_{\Re}^{2}+\phi_{\Im}^{2}\right\} .
\end{aligned}
$$

More specifically, $\Xi_{\phi}$ is linearly independent and

$$
\sup _{0 \not \equiv \in \operatorname{span}\left\{\Xi_{\phi}\right\}} \mu\left(\mathcal{Z}_{h} \cap(0,1)\right)=0,
$$

where $\mu$ is the Lebesgue measure and $\operatorname{span}\left\{\Xi_{\phi}\right\}$ is defined via (1.4). Clearly, (1.8) (a measure perspective) is essentially different from (1.6) (a cardinality perspective). Compared with the cardinality perspective, we will profit more from the measure perspective. Details on this will be given in section I-E. For simplicity we give the following definitions.

Definition 1.2: If (1.8) holds, then we say that the system $\Xi_{\phi}$ satisfies the generalized Haar condition (GHC for short), and $\phi$ is a complex-valued GHC-generator.

As a counterpart of Definition 1.2, we next define the GHC related to the real-valued generator $\varphi$ in (1.1).

Definition 1.3: If $\Lambda_{\varphi}=\{\varphi(\cdot+k): k=0, \ldots, s-1\}$ in (1.5) is linearly independent and satisfies

$$
\sup _{0 \not \equiv \in \operatorname{span}\left\{\Lambda_{\varphi}\right\}} \mu\left(\mathcal{Z}_{h} \cap(0,1)\right)=0,
$$

then we say that the system $\Lambda_{\varphi}$ satisfies the GHC, and $\varphi$ is a real-valued GHC-generator.

\section{Typical GHC-generators}

1) Typical complex-valued GHC-generators: We start with the amplitude-phase form of a complex-valued function. Any function (including a generator for an SIS) $F: \mathbb{R} \longrightarrow \mathbb{C}$ can be written as the general form $|F(t)| e^{\mathbf{i} \theta(F(t))}$, where $|F(t)|$ and $\theta(F(t))$ (taking values on $[0,2 \pi))$ are referred to as the amplitude function and the phase (or rotation) function. Therefore, any complexvalued generator for an SIS can be interpreted as the (possibly nonlinear) rotation of a real-valued function.
As mentioned before, one of the reasons for PR in optics is the high oscillation of a signal. Chirps which take the very general form $F(t) e^{\mathbf{i} \lambda \rho(t)}$ are the typical class of highly oscillatory signals, where $F(t) \geq 0$ and $\lambda$ is a (large) base frequency such that the phase function $\lambda \rho(t)$ is varying rapidly over time (c.f.[40]). As stated in [40], chirps are ubiquitous in nature. They are of interest in applications such as in analysis of echolocation in bats ([41]) and whales ([42], [40]), and in detecting gravitational waves ([40]). They are also applied in ultrafast optics ([43]) and ultrashort laser pulses ([44]).

Many chirps such as those in [34, section 6.3] have the local analytic structure. Employing this, GHC (1.8) can be easily checked. For example, motivated by [34, section 6.3] we take the chirp-generator

$$
\phi(x)=\frac{2}{3} \sqrt{2 \pi|b|} e^{-\mathbf{i} \frac{a(x-2)^{2}}{2 b}} e^{-\mathbf{i} \frac{p(x-2)}{b}} \cos ^{2} \frac{\pi(x-2)}{4} \chi_{(0,4)}(x),
$$

such that $\operatorname{supp}(\phi)=(0,4)$, where $a \neq 0$ and $\chi_{E}$ is the characteristic function of the set $E \subseteq \mathbb{R}$. Clearly, the 7 components of the system $\Xi_{\phi}$ in (1.7) are essentially the restrictions of analytic functions. Recall that the zero set of any nonzero analytic function has Lebesgue measure zero (c.f. [45]). Hence, if the components $g_{i} \in \Xi_{\phi}, i=$ $1, \ldots, 7$, are linearly independent on $(0,1)$ then $\phi$ is a complex-valued GHC-generator. The independence can be achieved if there exists $\left(x_{1}, \ldots, x_{7}\right) \in(0,1)^{7}$ such that the determinant

$$
\left|\begin{array}{cccc}
g_{1}\left(x_{1}\right) & g_{2}\left(x_{1}\right) & \ldots & g_{7}\left(x_{1}\right) \\
\vdots & \vdots & \ddots & \vdots \\
g_{1}\left(x_{7}\right) & g_{2}\left(x_{7}\right) & \ldots & g_{7}\left(x_{7}\right)
\end{array}\right| \neq 0
$$

Take the parameters $(a, b, p)=(4,0.8,1)$ or $(50,0.8,1)$ for example. Uniformly choosing $\left(x_{1}, \ldots, x_{7}\right)$ from $(0,1)^{7}$ we found that $(1.11)$ holds with probability 1 . Then $\phi$ in (1.10) is a complex-valued GHC-generator.

2) Real-valued GHC-generators: If a real-valued generator has the local analytic structure, then we can also address how to check whether it is a GHC-generator by the similar argument as above. The cardinal B-splines and the refinable functions ([46], [47], [48]) having positive masks are the typical examples of real-valued GHC-generators.

\section{Contributions}

An infinite discrete set $E$ is said to have sampling density (SD) if $\mathrm{SD}=\lim _{b-a \rightarrow \infty} \frac{\#([a, b] \cap E)}{b-a}<\infty$. Throughout this paper, we require that the random sampling points on any unit interval $[n, n+1]$ obey the uniform distribution. Our contributions include:

(i) From a new perspective-zero distribution, we establish the condition for the PLS of causal signals in the 
complex-generated SISs. Specifically, Theorem 2.5 will state that if $\phi$ is a complex-valued GHC-generator, then with probability 1 the random $\mathrm{SD}=3$ is sufficient for the PLS of nonseparable signals in $V_{\mathrm{ca}}(\phi)$.

(ii) The PLS of nonseparable and real-valued signals in real-generated SISs is also investigated from the zero distribution perspective. Specifically, Theorem 3.1 will state that if $\varphi$ is a real-valued GHC-generator, then with probability 1 the random SD $=2$ is sufficient for the PLS of nonseparable and real-valued signals in $V_{\mathrm{ca}}(\varphi)$.

(iii) An alternating approach, termed as phase decodingcoefficient recovery (PD-CR), is established to reconstruct the nonseparable signals in $V_{\mathrm{ca}}(\phi)$ and $V_{\mathrm{ca}}(\varphi)$. By the random sampling-based PD-CR, Propositions 2.6 and 3.2 will guarantee that the highly oscillatory signals can be locally reconstructed by using a very small number of samples. More details about this is given in section I-E-2).

\section{E. Highlights}

1) The zero distribution perspective enables us to do PLS in complex-generated $V_{c a}(\phi)$ : As mentioned in section I-B2, the traditional requirement-full spark property for PLS of real-valued signals can be interpreted by (1.6), a cardinality perspective. Theorem 1.1 implies that the property does not work for complex-valued case. Based on GHC (a measure perspective), we establish the PLS of nonseparable signals in $V_{\mathrm{ca}}(\phi)$.

2) Local reconstruction of highly oscillatory signals costs a small number of samples: If $\varphi$ or $\phi$ is highly oscillatory, then the quantities $\sup _{0 \neq h \in \operatorname{span}\left\{\Lambda_{\varphi}\right\}} \#\left(\mathcal{Z}_{h} \cap(0,1)\right)$ and $\sup _{0 \not k \in \operatorname{span}\left\{\Xi_{\phi}\right\}} \#\left(\mathcal{Z}_{h} \cap(0,1)\right)$ are great. And a great number of deterministic samples are necessary for local reconstruction. However, Propositions 2.6 and 3.2 will guarantee that the highly oscillatory signals can be locally reconstructed, with probability 1 , by using a very small number of random samples. Unlike [24], the number of samples is independent of the above quantities. To make this point, we will give a test signal in section III-C (3.46) and its local restriction in (3.47). Although the restriction is determined by just two coefficients, one needs at least 259 deterministic samples to reconstruct it. By the PD-CR, however, with probability 1 it can be reconstructed by just three random samples.

\section{F. Organization}

Section II concerns on the random PLS of nonseparable signals in $V_{\mathrm{ca}}(\phi)$, where $\phi$ is a complex-valued GHCgenerator. Based on the proposed PD-CR, we proved that when the sampling points obey the uniform distribution and the random $\mathrm{SD}=3$, then with probability 1 any nonseparable signal in $V_{\mathrm{ca}}(\phi)$ can be determined up to a unimodular scalar. In section III the PD-CR is modified such that it is more adaptive to the real-valued case. By the modified PD-CR, the real-valued and nonseparable signals in $V_{\mathrm{ca}}(\varphi)$ can be determined with probability 1 if the random $\mathrm{SD}=2$. To confirm our results numerical simulations are conducted in section II-H and section III-C. For the local reconstruction, Propositions 2.6 and 3.2 imply that the highly oscillatory signals can be determined, with probability 1 , by using a very small number of random samples. We conclude in section IV.

\section{RANDOM PLS OF CAUSAL SIGNALS IN COMPLEX-GENERATED SISS}

We start with some necessary denotations. As in section I the conjugate of $a \in \mathbb{C}$ is denoted by $\bar{a}$. The random variable $t$, obeying the uniform distribution on $(0,1)$, is denoted by $t \sim \mathbf{U}(0,1)$. Its observed value is denoted by $\widehat{t}$. For an event $\mathfrak{E}$, its probability and complementary event are denoted by $P(\mathfrak{E})$ and $\mathfrak{E}^{c}$, respectively. For two events $\mathfrak{E}_{1}$ and $\mathfrak{E}_{2}, P\left(\mathfrak{E}_{1} \cap \mathfrak{E}_{2}\right)=P\left(\mathfrak{E}_{1} \mid \mathfrak{E}_{2}\right) P\left(\mathfrak{E}_{2}\right)$, where $\mathfrak{E}_{1} \cap \mathfrak{E}_{2}$ and $P\left(\mathfrak{E}_{1} \mid \mathfrak{E}_{2}\right)$ are the intersection event and conditional probability, respectively.

\section{A. Preliminary on complex-valued GHC-generator}

The following proposition will be helpful for proving Theorem 2.5, one of our main theorems.

Proposition 2.1: Let $\phi=\phi_{\Re}+\mathbf{i} \phi_{\Im}$ be a GHC-generator supported on $(0, s)$. Then $\Lambda_{\phi, 1}:=\{\phi(\cdot+k): k=$ $0, \ldots, s-1\}$ and $\Lambda_{\phi, 2}:=\{\phi \bar{\phi}(\cdot+k): k=0, \ldots, s-1\}$ also satisfy the GHC, namely, (1.8) holds with $\Xi_{\phi}$ being replaced by $\Lambda_{\phi, 1}$ or $\Lambda_{\phi, 2}$.

Proof: The proof can be easily concluded by the GHC in (1.8) associated with $\Xi_{\phi}$.

Note 2.2: Theorem 1.1 implies that $\Lambda_{\phi, 1}$ satisfying GHC is not sufficient for achieving the PLS in $V_{\mathrm{ca}}(\phi)$. By the same argument as in the proof of Theorem 1.1, it is easy to prove that $\Lambda_{\phi, 2}$ is also not sufficient.

\section{B. Phase decoding-coefficient recovery for $V_{c a}(\phi)$}

As in section $\mathrm{I}, V_{\mathrm{ca}}(\phi)$ is defined by

$V_{\mathrm{ca}}(\phi)=\left\{\sum_{k=0}^{\infty} c_{k} \phi(\cdot-k):\left\{c_{k}\right\} \in \ell^{2}, c_{0} \neq 0, c_{k} \in \mathbb{C}\right\}$.

For $f \in V_{\mathrm{ca}}(\phi)$, call $\mathcal{N}_{f}:=\sup \left\{k: c_{k} \neq 0\right\}$ the maximum coefficient index of $f$. Clearly, if $f$ is compactly supported (infinitely supported) then $\mathcal{N}_{f}<\infty(=\infty)$. On the other hand, if the phases of sufficiently many samples of $|f|$ have been decoded, then the reconstruction of $f$ can be linear. Motivated by this, we will establish an alternating approach: phase decoding-coefficient recovery 
(PD-CR). Some denotations are necessary for introducing the approach.

As in section I, $\phi$ is supported on $(0, s)$ with the integer $s \geq 2$. For $n \geq 1$, define the set $I_{n}$ by

$$
I_{n}:= \begin{cases}\{0,1, \ldots, n-1\}, & 1 \leq n \leq s-1 \\ \{n-s+1, \ldots, n-1\}, & n \geq s .\end{cases}
$$

For $f=\sum_{k=0}^{\infty} c_{k} \phi(\cdot-k) \in V_{\mathrm{ca}}(\phi)$, define the auxiliary function $v_{n, f}$ on $(0,1)$ by

$$
v_{n, f}(\cdot):=\sum_{k \in I_{n}} c_{k} \phi(n+\cdot-k),
$$

which together with $\operatorname{supp}(\phi) \subseteq(0, s)$ leads to

$$
f(n+x)=v_{n, f}(x)+c_{n} \phi(x), x \in(0,1) .
$$

Based on $v_{n, f}$, define two auxiliary bivariate functions

$$
\begin{aligned}
& A_{n, f}(x, y)+B_{n, f}(x, y) \mathbf{i} \\
& :=\frac{|f|(n+x)}{|\phi|^{2}(x)}\left[\bar{\phi}(x) \phi(y) \bar{v}_{n}(y)-\bar{v}_{n}(x)|\phi|^{2}(y)\right],
\end{aligned}
$$

and

$$
\begin{aligned}
& C_{n, f}(x, y) \\
& :=|f|^{2}(n+y)-\left|v_{n, f}\right|^{2}(y)+2 \Re\left(\frac{v_{n, f}(x) \bar{v}_{n, f}(y) \phi(y)}{\phi(x)}\right) \\
& -\frac{|\phi|^{2}(y)}{|\phi|^{2}(x)}\left[|f|^{2}(n+x)+\left|v_{n, f}\right|^{2}(x)\right],
\end{aligned}
$$

whenever $x, y \in(0,1)$ such that $\phi(x) \neq 0$. The values of the above bivariate functions at $(x, y) \in(0,1)^{2}$ are correlated via the following equation w.r.t the unknown $z \in \mathbb{C}$ :

$$
\begin{aligned}
& \left(A_{n, f}(x, y)+B_{n, f}(x, y) \mathbf{i}\right) z^{2}-C_{n, f}(x, y) z \\
& +A_{n, f}(x, y)-B_{n, f}(x, y) \mathbf{i}=0 .
\end{aligned}
$$

The following lemma states that the solutions to (2.17) can provide a precise feedback on the global phase of $\left\{c_{k}\right\}_{k \in I_{n}}$.

Lemma 2.3: Let $v_{n, f}(\cdot)$ and $A_{n, f}(\cdot, \cdot)+B_{n, f}(\cdot, \cdot)$ i be defined in (2.13) and (2.15), respectively. Define $\widetilde{v}_{n, f}(\cdot)$ via (2.13) with $\left\{c_{k}\right\}_{k \in I_{n}}$ being replaced by $\left\{\widetilde{c}_{k}\right\}_{k \in I_{n}}:=$ $e^{\mathbf{i} \widehat{\theta}}\left\{c_{k}\right\}_{k \in I_{n}}$. Moreover, define $\widetilde{A}_{n, f}(\cdot, \cdot)+\widetilde{B}_{n, f}(\cdot, \cdot) \mathbf{i}$ and $\widetilde{C}_{n, f}(\cdot, \cdot)$ via (2.15) and (2.16) with $v_{n, f}(\cdot)$ being replaced by $\widetilde{v}_{n, f}(\cdot)$. For fixed $x, y \in(0,1)$ and $n \geq 1$ such that $\phi(x) \neq 0$ and $A_{n, f}(x, y)+B_{n, f}(x, y) \mathbf{i} \neq 0$, suppose that the two solutions to (2.17) are $z_{1}$ and $z_{2}$. Then the two solutions to

$$
\begin{aligned}
& \left(\widetilde{A}_{n, f}(x, y)+\widetilde{B}_{n, f}(x, y) \mathbf{i}\right) z^{2}-\widetilde{C}_{n, f}(x, y) z \\
& +\widetilde{A}_{n, f}(x, y)-\widetilde{B}_{n, f}(x, y) \mathbf{i}=0
\end{aligned}
$$

are $e^{\mathbf{i} \widehat{\theta}} z_{1}$ and $e^{\mathbf{i} \widehat{\theta}} z_{2}$
Proof: Through the direct calculation we have $\frac{v_{n, f}(x) \bar{v}_{n, f}(y) \phi(y)}{\phi(x)}=\frac{\widetilde{v}_{n, f}(x) \overline{\tilde{v}}_{n, f}(y) \phi(y)}{\phi(x)}$ and

$$
C_{n, f}(x, y)=\widetilde{C}_{n, f}(x, y), \frac{\widetilde{A}_{n, f}(x, y)+\widetilde{B}_{n, f}(x, y) \mathbf{i}}{A_{n, f}(x, y)+B_{n, f}(x, y) \mathbf{i}}=e^{-\mathbf{i} \widehat{\theta}}
$$

On the other hand,

$$
\begin{aligned}
& z_{1}, z_{2} \\
& =\frac{C_{n, f}(x, y) \pm \sqrt{C_{n, f}^{2}(x, y)-4\left|A_{n, f}(x, y)+B_{n, f}(x, y) \mathbf{i}\right|^{2}}}{2\left(A_{n, f}(x, y)+B_{n, f}(x, y) \mathbf{i}\right)},
\end{aligned}
$$

which together with (2.19) leads to that the two solutions to (2.18) are $e^{\mathbf{i} \widehat{\theta}} z_{1}$ and $e^{\mathbf{i} \widehat{\theta}} z_{2}$.

Based on Lemma 2.3, the following theorem concerns on a guarantee for decoding phases.

Theorem 2.4: Let $f \in V_{\text {ca }}(\phi)$. Assume that all the samples

$$
\begin{aligned}
\left\{\left|f\left(\widehat{t}_{0}\right)\right|\right\} \cup\left\{\left|f\left(n+\widehat{t}_{n_{j}}\right)\right|:\right. & j=1,2,3, n=1, \ldots, \\
& \left.\mathcal{N}_{f}+s-1\right\}
\end{aligned}
$$

are nonzeros where $\widehat{t}_{0}, \widehat{t}_{n_{j}} \in(0,1)$. Then the corresponding phases $\left\{\theta\left(f\left(\widehat{t}_{0}\right)\right)\right\} \cup\left\{\theta\left(f\left(n+\widehat{t}_{n_{j}}\right)\right)\right\}_{n, j}$ can be determined (up to a global real-valued number) if for every $n \in\left\{1,2, \ldots, \mathcal{N}_{f}+s-1\right\}, \phi\left(\widehat{t}_{n_{1}}\right) \neq 0$ and the equation system

$$
\left\{\begin{array}{l}
\left(A_{n, f}\left(\widehat{t}_{n_{1}}, \widehat{t}_{n_{2}}\right)+B_{n, f}\left(\widehat{t}_{n_{1}}, \widehat{t}_{n_{2}}\right) \mathbf{i}\right) z^{2}-C_{n, f}\left(\widehat{t}_{n_{1}}, \widehat{t}_{n_{2}}\right) z \\
+A_{n, f}\left(\widehat{t}_{n_{1}}, \widehat{t}_{n_{2}}\right)-B_{n, f}\left(\widehat{t}_{n_{1}}, \widehat{t}_{n_{2}}\right) \mathbf{i}=0, \\
\left(A_{n, f}\left(\widehat{t}_{n_{1}}, \widehat{t}_{n_{3}}\right)+B_{n, f}\left(\widehat{t}_{n_{1}}, \widehat{t}_{n_{3}}\right) \mathbf{i}\right) z^{2}-C_{n, f}\left(\widehat{t}_{n_{1}}, \widehat{t}_{n_{3}}\right) z \\
+A_{n, f}\left(\widehat{t}_{n_{1}}, \widehat{t}_{n_{3}}\right)-B_{n, f}\left(\widehat{t}_{n_{1}}, \widehat{t}_{n_{3}}\right) \mathbf{i}=0,
\end{array}\right.
$$

w.r.t the the unknown $z \in \mathbb{C}$ has a unique solution.

Proof: As previously, denote $f=\sum_{k=0}^{\infty} c_{k} \phi(\cdot-k)$. We prove the theorem recursively on $n$. Suppose that

$$
\theta\left(f\left(\widehat{t}_{0}\right)\right)=\theta_{0}
$$

is known as the priori information. Then it follows from $0 \neq f\left(\widehat{t}_{0}\right)=c_{0} \phi\left(\widehat{t}_{0}\right)$ that

$$
c_{0}=e^{\mathbf{i} \theta_{0}}\left|f\left(\widehat{t}_{0}\right)\right| / \phi\left(\widehat{t}_{0}\right) .
$$

For $n=1$, we next address how to determine $z:=$ $e^{\mathbf{i} \theta\left(f\left(\widehat{t}_{1}+1\right)\right)}$. It follows from (2.14) that

$$
\left\{\begin{aligned}
\left|v_{1, f}\left(\widehat{t}_{1_{1}}\right)+c_{1} \phi\left(\widehat{t}_{1_{1}}\right)\right| & =\left|f\left(1+\widehat{t}_{1_{1}}\right)\right|, \\
\left|v_{1, f}\left(\widehat{t}_{1_{2}}\right)+c_{1} \phi\left(\widehat{t}_{1_{2}}\right)\right| & =\left|f\left(1+\widehat{t}_{1_{2}}\right)\right|, \\
\left|v_{1, f}\left(\widehat{t}_{1_{3}}\right)+c_{1} \phi\left(\widehat{t}_{1_{3}}\right)\right| & =\left|f\left(1+\widehat{t}_{1_{3}}\right)\right|,
\end{aligned}\right.
$$

where $v_{1, f}\left(\widehat{t}_{1_{j}}\right), j=1,2,3$, are computed by using (2.13) and (2.23) as follows,

$$
v_{1, f}\left(\widehat{t}_{1_{j}}\right)=\phi\left(1+\widehat{t}_{1_{j}}\right) c_{0}=\frac{\phi\left(1+\widehat{t}_{1_{j}}\right) e^{\mathbf{i} \theta_{0}}\left|f\left(\widehat{t}_{0}\right)\right|}{\phi\left(\widehat{t}_{0}\right)} .
$$


By (2.14), $\left|f\left(1+\widehat{t}_{1_{1}}\right)\right| z=v_{1, f}\left(\widehat{t}_{1_{1}}\right)+c_{1} \phi\left(\widehat{t}_{1_{1}}\right)$. Since $\phi\left(\widehat{t}_{1_{1}}\right) \neq 0$, we have

$$
c_{1}=\frac{\left|f\left(1+\widehat{t}_{1_{1}}\right)\right| z-v_{1, f}\left(\widehat{t}_{1_{1}}\right)}{\phi\left(\widehat{t}_{1_{1}}\right)},
$$

which together with the last two identities in (2.24) leads to

$$
\begin{aligned}
& \left|v_{1, f}\left(\widehat{t}_{1_{j}}\right)+\frac{\left|f\left(1+\widehat{t}_{1_{1}}\right)\right| z-v_{1, f}\left(\widehat{t}_{1_{1}}\right)}{\phi\left(\widehat{t}_{1_{1}}\right)} \phi\left(\widehat{t}_{1_{j}}\right)\right|^{2} \\
& =\left|f\left(1+\widehat{t}_{1_{j}}\right)\right|^{2}, j=2,3 .
\end{aligned}
$$

By direct calculation, we can prove that (2.27) is equivalent to (2.21) for $n=1$. Since there exists a unique solution to (2.21), $z$ can be determined, and consequently $c_{1}$ can be done by (2.26). Now $\left\{\theta\left(f\left(1+\widehat{t}_{1_{l}}\right)\right): l \neq 1\right\}$ are determined by $\theta\left(f\left(1+\widehat{t}_{1_{l}}\right)\right)=\theta\left(v_{1, f}\left(\widehat{t}_{1_{l}}\right)+c_{1} \phi\left(\widehat{t}_{1_{l}}\right)\right)$ with $v_{1, f}\left(\widehat{t}_{1_{l}}\right)$ given by (2.25). Suppose that $\left\{\theta\left(f\left(\widehat{t}_{0}\right)\right)\right\} \cup$ $\left\{\theta\left(f\left(k+\widehat{t}_{k_{j}}\right)\right): j=1,2,3, k=1, \ldots, n-1\right\}$ and $\left\{c_{k}\right\}_{k=0}^{n-1}$ have been determined, where $n<\mathcal{N}_{f}+s$. Through the similar procedures as above, $z:=e^{\mathbf{i} \theta\left(f\left(\widehat{t}_{n_{1}}+n\right)\right)}$ can be determined by (2.21), and $c_{n}=\left[\left|f\left(n+\widehat{t}_{n_{1}}\right)\right| z-\right.$ $\left.v_{n, f}\left(\widehat{t}_{n_{1}}\right)\right] / \phi\left(\widehat{t}_{n_{1}}\right)$. Then by (2.14), $\theta\left(f\left(n+\widehat{t}_{n_{j}}\right)\right)$ can be computed. By the recursion on $n$, the phases $\left\{\theta\left(f\left(\widehat{t}_{0}\right)\right)\right\} \cup$ $\left\{\theta\left(f\left(n+\widehat{t}_{n_{j}}\right)\right): j=1,2,3, n=1, \ldots, \mathcal{N}_{f}+s-1\right\}$ can be determined.

Recall that the above determination is achieved by the priori information (2.22). Without this information, now we assign

$$
\theta\left(f\left(\widehat{t}_{0}\right)\right)=\widetilde{\theta}_{0}
$$

where $\widetilde{\theta}_{0} \in[0,2 \pi)$. We next prove that under this assignment, $\tilde{f}:=e^{\mathbf{i}\left(\widetilde{\theta}_{0}-\theta_{0}\right)} f=\sum_{k=0}^{\infty} \widetilde{c}_{k} \phi(\cdot-k)$ can be determined by the samples in (2.20), where $\widetilde{c}_{k}=e^{\mathbf{i}\left(\widetilde{\theta}_{0}-\theta_{0}\right)} c_{k}$. Consequently, $\left\{\theta\left(f\left(\widehat{t}_{0}\right)\right)+\widetilde{\theta}_{0}-\theta_{0}\right\} \cup\left\{\theta\left(f\left(n+\widehat{t}_{n_{j}}\right)\right)+\widetilde{\theta}_{0}-\right.$ $\left.\theta_{0}: j=1,2,3, n=1, \ldots, \mathcal{N}_{f}+s-1\right\}$ can be determined.

For (2.28), through the similar analysis as in (2.23) we have

$$
\widetilde{c}_{0}=\frac{e^{\mathbf{i} \widetilde{\theta}_{0}}\left|f\left(\widehat{t}_{0}\right)\right|}{\phi\left(\widehat{t}_{0}\right)}=e^{\mathbf{i}\left(\widetilde{\theta}_{0}-\theta_{0}\right)} c_{0}
$$

and $\theta\left(\widetilde{f}\left(\widehat{t}_{0}\right)\right)=\theta\left(\phi\left(\widehat{t}_{0}\right) \widetilde{c}_{0}\right)=\theta\left(f\left(\widehat{t}_{0}\right)\right)+\widetilde{\theta}_{0}-\theta_{0}$. As in Lemma 2.3, define $\widetilde{A}_{1, f}(x, y)+\widetilde{B}_{1, f}(x, y) \mathbf{i}$ and $\widetilde{C}_{1, f}(x, y)$ via (2.15) and (2.16) with $c_{0}$ being replaced by $\widetilde{c}_{0}$. Through the similar analysis as in (2.27), $z:=$ $e^{\mathbf{i} \theta\left(\widetilde{f}\left(\widehat{t}_{1}+1\right)\right)}$ satisfies

$$
\left\{\begin{array}{l}
\left(\widetilde{A}_{1, f}\left(\widehat{t}_{1_{1}}, \widehat{t}_{1}\right)+\widetilde{B}_{1, f}\left(\widehat{t}_{1_{1}}, \widehat{t}_{1_{2}}\right) \mathbf{i}\right) z^{2}-\widetilde{C}_{1, f}\left(\widehat{t}_{1_{1}}, \widehat{t}_{1_{2}}\right) z \\
+\widetilde{A}_{1, f}\left(\widehat{t}_{1_{1}}, \widehat{t}_{1_{2}}\right)-\widetilde{B}_{1, f}\left(\widehat{t}_{1_{1}}, \widehat{t}_{1_{2}}\right) \mathbf{i}=0 \\
\left(\widetilde{A}_{1, f}\left(\widehat{t}_{1_{1}}, \widehat{t}_{1_{3}}\right)+\widetilde{B}_{1, f}\left(\widehat{t}_{1_{1}}, \widehat{t}_{1_{3}}\right) \mathbf{i}\right) z^{2}-\widetilde{C}_{1, f}\left(\widehat{t}_{1_{1}}, \widehat{t}_{1_{3}}\right) z \\
+\widetilde{A}_{1, f}\left(\widehat{t}_{1_{1}}, \widehat{t}_{1_{3}}\right)-\widetilde{B}_{1, f}\left(\widehat{t}_{1_{1}}, \widehat{t}_{1_{3}}\right) \mathbf{i}=0 .
\end{array}\right.
$$

By the same argument as in the proof of Lemma 2.3, we can prove that

$$
\frac{\widetilde{A}_{1, f}(x, y)+\widetilde{B}_{1, f}(x, y) \mathbf{i}}{A_{1, f}(x, y)+B_{1, f}(x, y) \mathbf{i}}=e^{\mathbf{i}\left(\theta_{0}-\widetilde{\theta}_{0}\right)}, \widetilde{C}_{1, f}(x, y)=C_{1, f}(x, y),
$$

which together with (2.21) having a unique solution leads to that (2.29) also has a unique solution. Applying Lemma 2.3 with $\widehat{\theta}=\widetilde{\theta}_{0}-\theta_{0}$, we have $z=e^{\mathbf{i}\left(\widetilde{\theta}_{0}-\theta_{0}\right)} e^{\mathbf{i} \theta\left(f\left(\widehat{t}_{1}+1\right)\right)}$. Consequently, $\widetilde{c}_{1}=e^{\mathbf{i}\left(\widetilde{\theta}_{0}-\theta_{0}\right)} c_{1}$. Suppose that $\left\{\theta\left(f\left(\widehat{t}_{0}\right)\right)+\right.$ $\left.\widetilde{\theta}_{0}-\theta_{0}\right\} \cup\left\{\theta\left(f\left(k+\widehat{t}_{k_{j}}\right)\right)+\widetilde{\theta}_{0}-\theta_{0}: j=1,2,3, k=\right.$ $1, \ldots, n-1\}$ (or $\widetilde{c}_{k}=e^{\mathbf{i}\left(\widetilde{\theta}_{0}-\theta_{0}\right)} c_{k}$ ) have been determined. Define $\widetilde{A}_{n, f}+\widetilde{B}_{n, f} \mathbf{i}$ and $\widetilde{C}_{n, f}$ via (2.15) and (2.16), respectively, by replacing $c_{k}$ by $\widetilde{c}_{k}$. By Lemma 2.3 and the similar analysis as in (2.30), we can prove that $\widetilde{c}_{n}=e^{\mathbf{i}\left(\widetilde{\theta}_{0}-\theta_{0}\right)} c_{n}$. The rest of the proof can be easily concluded by the recursion on $n$.

The procedures in the proof of Theorem 2.4 for decoding the phases $\left\{\theta\left(f\left(\widehat{t}_{0}\right)\right)\right\} \cup\left\{\theta\left(f\left(n+\widehat{t}_{n_{j}}\right)\right)\right\}_{n, j}$, up to a global real number, are conducted recursively on $n$. And they alternate with those for recovering the coefficients $\left\{c_{n}\right\}$. Next we summarize them to establish the PD-CR.

\section{Approach II-B}

Input: Samples $\left\{\left|f\left(\widehat{t}_{0}\right)\right|\right\} \cup\left\{\left|f\left(k+\widehat{t}_{k_{j}}\right)\right|: j=\right.$ $1,2,3, k=1, \ldots, n\}$ where $\widehat{t}_{0}, \widehat{t}_{k_{j}} \in(0,1)$ and $n<\mathcal{N}_{f}+$ $s$; initial phase $\theta\left(f\left(\widehat{t}_{0}\right)\right)=\widetilde{\theta}_{0}$ and $c_{0}:=e^{\mathbf{i} \widetilde{\theta}_{0}}\left|f\left(\widehat{t}_{0}\right)\right| / \phi\left(\widehat{t}_{0}\right)$.

Output: $\left\{c_{k}\right\}_{k=0}^{n}$ and $\left\{\theta\left(f\left(\widehat{t}_{0}\right)\right)\right\} \cup\left\{\theta\left(f\left(k+\widehat{t}_{k_{j}}\right)\right): j=\right.$ $1,2,3, k=1, \ldots, n\}$.

Recursion assumption: Assume that the phases $\left\{\theta\left(f\left(\widehat{t}_{0}\right)\right)\right\} \cup\left\{\theta\left(f\left(k+\widehat{t}_{k_{j}}\right)\right): j=1,2,3, k=1, \ldots, n-\right.$ $1\}$ and coefficients $\left\{c_{k}\right\}_{k=0}^{n-1}$ have been recovered. Then $\left\{\theta\left(f\left(n+\widehat{t}_{n_{j}}\right)\right): j=1,2,3\right\}$ and $c_{n}$ are recovered by the following steps:

step 1: Compute $v_{n, f}\left(\widehat{t}_{n_{j}}\right)$ via (2.13) with $j=1,2,3$. Compute $A_{n, f}\left(\widehat{t}_{n_{1}}, \widehat{t}_{n_{2}}\right)+B_{n, f}\left(\widehat{t}_{n_{1}}, \widehat{t}_{n_{2}}\right) \mathbf{i}$ and $A_{n, f}\left(\widehat{t}_{n_{1}}, \widehat{t}_{n_{3}}\right)+B_{n, f}\left(\hat{t}_{n_{1}}, \widehat{t}_{n_{3}}\right) \mathbf{i}, \quad C_{n, f}\left(\hat{t}_{n_{1}}, \hat{t}_{n_{2}}\right) \quad$ and $C_{n, f}\left(\widehat{t}_{n_{1}}, \widehat{t}_{n_{3}}\right)$ via (2.15) and (2.16), respectively.

step 2: $\theta\left(f\left(n+\widehat{t}_{n_{1}}\right)\right)$ is decoded by computing

$$
\begin{aligned}
& e^{\mathbf{i} \theta\left(f\left(n+\widehat{t}_{n_{1}}\right)\right)} \\
& =\arg \min _{z_{n, k} \in\left\{z_{n, 1}, z_{n, 2}\right\}}\left\{\min \left\{\left|z_{n, k}-z_{n, l}\right|: l=3,4\right\}\right\}
\end{aligned}
$$

where

$$
\begin{aligned}
& z_{n, k} \\
& =\frac{C_{n, f}\left(\widehat{t}_{n_{1}}, \widehat{t}_{n_{2}}\right)}{2\left(A_{n, f}\left(\widehat{t}_{n_{1}}, \widehat{t}_{n_{2}}\right)+B_{n, f}\left(\widehat{t}_{n_{1}}, \widehat{t}_{n_{2}}\right) \mathbf{i}\right)} \\
& \pm \frac{\sqrt{C_{n, f}^{2}\left(\widehat{t}_{n_{1}}, \widehat{t}_{n_{2}}\right)-4\left|A_{n, f}\left(\widehat{t}_{n_{1}}, \widehat{t}_{n_{2}}\right)+B_{n, f}\left(\widehat{t}_{n_{1}}, \widehat{t}_{n_{2}}\right) \mathbf{i}\right|^{2}}}{2\left(A_{n, f}\left(\widehat{t}_{n_{1}}, \widehat{t}_{n_{2}}\right)+B_{n, f}\left(\widehat{t}_{n_{1}}, \widehat{t}_{n_{2}}\right) \mathbf{i}\right)}
\end{aligned}
$$


with $k=1,2$, and

$$
\begin{aligned}
& z_{n, l} \\
& =\frac{C_{n, f}\left(\widehat{t}_{n_{1}}, \widehat{t}_{n_{3}}\right)}{2\left(A_{n, f}\left(\widehat{t}_{n_{1}}, \widehat{t}_{n_{3}}\right)+B_{n, f}\left(\widehat{t}_{n_{1}}, \widehat{t}_{n_{3}}\right) \mathbf{i}\right)} \\
& \pm \frac{\sqrt{C_{n, f}^{2}\left(\widehat{t}_{n_{1}}, \widehat{t}_{n_{3}}\right)-4\left|A_{n, f}\left(\widehat{t}_{n_{1}}, \widehat{t}_{n_{3}}\right)+B_{n, f}\left(\widehat{t}_{n_{1}}, \widehat{t}_{n_{3}}\right) \mathbf{i}\right|^{2}}}{2\left(A_{n, f}\left(\widehat{t}_{n_{1}}, \widehat{t}_{n_{3}}\right)+B_{n, f}\left(\widehat{t}_{n_{1}}, \widehat{t}_{n_{3}}\right) \mathbf{i}\right)}
\end{aligned}
$$

with $l=3,4$.

step 3: Compute $c_{n}=\left[e^{\mathbf{i} \theta\left(f\left(n+\widehat{t}_{n_{1}}\right)\right)}\left|f\left(n+\widehat{t}_{n_{1}}\right)\right|-\right.$ $\left.v_{n, f}\left(\widehat{t}_{n_{1}}\right)\right] / \phi\left(\widehat{t}_{n_{1}}\right)$. Compute $f\left(n+\widehat{t}_{n_{j}}\right)$ by (2.14), and $\theta\left(f\left(n+\widehat{t}_{n_{j}}\right)\right)=\theta\left(\frac{f\left(n+\widehat{t}_{n_{j}}\right)}{\left|f\left(n+\widehat{t}_{n_{j}}\right)\right|}\right)$ where $j \neq 1$.

\section{Random phaseless sampling for $V_{c a}(\phi)$}

Next we replace the points $\left\{\widehat{t}_{0}\right\} \cup\left\{\widehat{t}_{n_{1}}, \widehat{t}_{n_{2}}, \widehat{t}_{n_{3}}\right\}_{n}$ in Theorem 2.4 (2.20) by random variables, and establish our first main theorem as follows.

Theorem 2.5: Let $\phi=\phi_{\Re}+\mathbf{i} \phi_{\Im}$ be a complex-valued GHC-generator such that $\operatorname{supp}(\phi) \subseteq(0, s)$ with the integer $s \geq 2$. Then any nonseparable signal $f \in V_{\text {ca }}(\phi)$ can be determined (up to a unimodular scalar) with probability 1 by the random samples $\left\{\left|f\left(t_{0}\right)\right|\right\} \cup\left\{\left|f\left(n+t_{n_{1}}\right)\right|, \mid f(n+\right.$ $\left.\left.t_{n_{2}}\right)|| f,\left(n+t_{n_{3}}\right) \mid: n=1, \ldots, \infty\right\}$, where the i.i.d random variables $\left\{t_{0}\right\} \cup\left\{t_{n_{1}}, t_{n_{2}}, t_{n_{3}}: n=1, \ldots, \infty\right\} \sim \mathbf{U}(0,1)$. Proof: The proof is given in section II-F.

The following proposition concerns on local reconstruction.

Proposition 2.6: Let $\phi=\phi_{\Re}+\mathbf{i} \phi_{\Im}$ and $f$ be as in Theorem 2.5. Then for any integer $L>1$, the restriction $f_{[0, L]}$ of $f$ on $[0, L]$ can be determined (up to a unimodular scalar) with probability 1 , by the random samples $\left\{\left|f\left(t_{0}\right)\right|\right\} \cup\left\{\left|f\left(n+t_{n_{1}}\right)\right|,\left|f\left(n+t_{n_{2}}\right)\right|,\left|f\left(n+t_{n_{3}}\right)\right|\right.$ : $n=1, \ldots, L-1\}$, where the i.i.d random variables $\left\{t_{0}\right\} \cup\left\{t_{n_{1}}, t_{n_{2}}, t_{n_{3}}: n=1, \ldots, L-1\right\} \sim \mathbf{U}(0,1)$. Proof: The proof is given in section II-G.

\section{Conjugation ambiguity does not occur for $V_{c a}(\phi)$}

In this section we will compare the result in section II-C with that in [29] which concerns on the conjugation phase retrieval. We start with the definition of conjugation ambiguity (c.f. [29], [14]) of PR in an SIS. The conjugation ambiguity means that there exists a signal $f$ in an SIS, which is not real-valued (up to a unimodular scalar), such that it is not distinguishable from its conjugation $\bar{f}$ which still lies in this SIS. For a real-valued generator $\varphi$, it is clear that

$$
\left|\sum_{k=0}^{N} c_{k} \varphi(\cdot-k)\right|=\left|\sum_{k=0}^{N} \bar{c}_{k} \varphi(\cdot-k)\right|
$$

for any sequence $\left\{c_{k}\right\}_{k=0}^{N} \subseteq \mathbb{C}$. That is, the conjugation ambiguity is inevitable for phaseless sampling in a realgenerated SIS. Most recently, C.K. Lai, F. Littmann and E. Weber [29] investigated the conjugate phase retrieval of complex-valued bandlimited signals, namely, to reconstruct them up to the conjugation ambiguity.

It is easy to check that (1.3) leads to (2.32). Despite all this, the following remark states that there are some essential differences between the result in Theorem 2.5 and that in [29].

Remark 2.7: (1) Unlike the conjugate phase retrieval, the conjugation ambiguity does not occur in Theorem 2.5. Or else, suppose that $f(x)=|f(x)| e^{\mathbf{i} \rho(x)}$ and $\bar{f}(x)=$ $|f(x)| e^{-\mathbf{i} \rho(x)}$ both lie in $V_{\text {ca }}(\phi)$. Clearly, $|f(x)|=|\bar{f}(x)|$. Then it follows from Theorem 2.5 that $f(x)=e^{i c} \bar{f}(x)$ which leads to $\rho(x) \equiv c / 2$. This is a contradiction with the definition of conjugation ambiguity. (2) Our generator is complex-valued and compactly supported while the generator in [29] is real-valued and not compactly-supported. (3) Our sampling is random while that in [29] is deterministic.

\section{E. Some lemmas for proving Theorem 2.5}

We start with the so called maximum gap.

Definition 2.8: For $f=\sum_{k=0}^{\infty} c_{k} \phi(\cdot-k) \in V_{\mathrm{ca}}(\phi)$, its maximum gap is defined as

$\mathcal{G}_{f}=\left\{\begin{array}{c}\max \left\{1 \leq \gamma<\infty: \exists i \geq 1 \text { s.t. } c_{i+\gamma} \neq 0,\right. \\ \left.c_{i}=\ldots=c_{i+\gamma-1}=0\right\}, \text { if } \exists c_{k}=0 \\ \text { else. }\end{array}\right.$

The following lemma concerns on the relationship between the maximum gap and nonseparability.

Lemma 2.9: If $f=\sum_{k=0}^{\infty} c_{k} \phi(\cdot-k) \in V_{\text {ca }}(\phi)$ is nonseparable, then $\mathcal{G}_{f}<s-1$.

Proof: Suppose that $0=c_{i}=\ldots=c_{i+L-1}$ with $i \geq 1$ and $L \geq s-1$. Define $f_{1}=\sum_{k=0}^{i-1} c_{k} \phi(\cdot-k)$ and $f_{2}=$ $\sum_{k=i+L}^{\infty} c_{k} \phi(\cdot-k)$. It is easy to derive from $c_{0} \neq 0$, $c_{i+L} \neq 0$ and $\phi$ being a GHC-generator that $f_{1} \neq \equiv 0, f_{2} \not \equiv$ 0 . Now $\operatorname{supp}(\phi) \subseteq(0, s)$ leads to $f=f_{1}+f_{2}$ and $f_{1} f_{2} \equiv$ 0 . That is, $f$ is separable. This is a contradiction.

The following lemma concerns on the zero property of $A_{n, f}(x, y)+B_{n, f}(x, y) \mathbf{i}$.

Lemma 2.10: Let $\phi=\phi_{\Re}+\mathbf{i} \phi_{\Im}$ be a complex-valued GHC-generator such that $\operatorname{supp}(\phi) \subseteq(0, s)$ with the integer $s \geq 2$. Moreover, $f \in V_{\mathrm{ca}}(\phi)$ is nonseparable, and $\left\{t_{0}\right\} \cup$ $\left\{t_{n_{1}}, t_{n_{2}}, t_{n_{3}}: n=1, \ldots, \mathcal{N}_{f}+s-1\right\} \sim \mathbf{U}(0,1)$. Then $P\left(A_{n, f}\left(t_{n_{1}}, t_{n_{i}}\right)+B_{n, f}\left(t_{n_{1}}, t_{n_{i}}\right) \mathbf{i} \neq 0\right)=1$ for any $n \in$ $\left\{1, \ldots, \mathcal{N}_{f}+s-1\right\}$, where $i=2,3$.

Proof: The proof is given in section V-A. 
Based on Lemma 2.10, in what follows we investigate the probabilistic behavior of the phase $\theta\left(A_{n, f}\left(t_{n_{1}}, t_{n_{2}}\right)\right.$ $\left.+\mathbf{i} B_{n, f}\left(t_{n_{1}}, t_{n_{2}}\right)\right)$.

Lemma 2.11: Let $f, \phi$ and $\left\{t_{0}\right\} \cup\left\{t_{n_{1}}, t_{n_{2}}, t_{n_{3}}: n=\right.$ $\left.1, \ldots, \mathcal{N}_{f}+s-1\right\} \sim \mathbf{U}(0,1)$ be as in Lemma 2.10. Then for any fixed $\alpha \in(0,2 \pi]$, it holds that

$$
P\left(\theta\left(A_{n, f}\left(t_{n_{1}}, t_{n_{2}}\right)+\mathbf{i} B_{n, f}\left(t_{n_{1}}, t_{n_{2}}\right)\right) \neq \alpha\right)=1 .
$$

Proof: The proof is given in section V-B.

Based on Lemma 2.11, we next investigate the uniqueness of (2.21) with $\widehat{t}_{n_{i}}$ therein being replaced by $t_{n_{i}} \sim$ $\mathbf{U}(0,1)$.

Lemma 2.12: Let $f, \phi$ and $\left\{t_{0}\right\} \cup\left\{t_{n_{1}}, t_{n_{2}}, t_{n_{3}}: n=\right.$ $\left.1, \ldots, \mathcal{N}_{f}+s-1\right\} \sim \mathbf{U}(0,1)$ be as in Lemma 2.10. Then for any $n \in\left\{1, \ldots, \mathcal{N}_{f}+s-1\right\}$, the equation system

$\left\{\begin{array}{l}\left(A_{n, f}\left(t_{n_{1}}, t_{n_{2}}\right)+B_{n, f}\left(t_{n_{1}}, t_{n_{2}}\right) \mathbf{i}\right) z^{2}-C_{n, f}\left(t_{n_{1}}, t_{n_{2}}\right) z \\ +A_{n, f}\left(t_{n_{1}}, t_{n_{2}}\right)-B_{n, f}\left(t_{n_{1}}, t_{n_{2}}\right) \mathbf{i}=0, \\ \left(A_{n, f}\left(t_{n_{1}}, t_{n_{3}}\right)+B_{n, f}\left(t_{n_{1}}, t_{n_{3}}\right) \mathbf{i}\right) z^{2}-C_{n, f}\left(t_{n_{1}}, t_{n_{3}}\right) z \\ +A_{n, f}\left(t_{n_{1}}, t_{n_{3}}\right)-B_{n, f}\left(t_{n_{1}}, t_{n_{3}}\right) \mathbf{i}=0,\end{array}\right.$ has only one solution with probability 1 .

Proof: The proof is given in section V-C.

Based on Lemma 2.12 and Approach II-B, we next prove Theorem 2.5.

\section{F. Proof of Theorem 2.5}

By $\Lambda_{\phi, 1}$ in Proposition 2.1 satisfying GHC, we have $P\left(\left|f\left(n+t_{n_{1}}\right)\right| \neq 0\right)=P\left(\left|\phi\left(t_{n_{1}}\right)\right| \neq 0\right)=1$. If the phases $\left\{\theta\left(f\left(t_{0}\right)\right)\right\} \cup\left\{\theta\left(f\left(n+t_{n_{i}}\right)\right): n=1, \ldots, \infty, i=1,2,3\right\}$ can be determined (up to a global real number) with probability 1 , then $\left\{c_{n}\right\}_{n=0}^{\infty}$ can be reconstructed by (2.14) (up to a unimodular scalar) with the same probability. On the other hand, by $\operatorname{supp}(\phi) \subseteq(0, s)$ and the definition of $\mathcal{N}_{f}$, it is sufficient to prove that $\left\{\theta\left(f\left(t_{0}\right)\right)\right\} \cup\{\theta(f(n+$ $\left.\left.\left.t_{n_{i}}\right)\right): n=1, \ldots, \mathcal{N}_{f}+s-1, i=1,2,3\right\}$ can be determined (up to a global real number) with probability 1.

As previously, we have $P\left(\left|\phi\left(t_{0}\right)\right| \neq 0\right)=1$. Following Approach II-B, let $c_{0}:=e^{\mathbf{i} \widehat{\theta}_{0}}\left|f\left(t_{0}\right)\right| / \phi\left(t_{0}\right)$. Then, with probability $1, c_{0}$ can be determined up to the scalar $e^{\mathbf{i} \widehat{\theta}}$, where $\widehat{\theta}:=\widetilde{\theta}_{0}-\theta_{0}$ with $\theta_{0}$ being the exact phase of $f\left(t_{0}\right)$. Or with probability $1, \theta\left(f\left(t_{0_{i}}\right)\right)$ can be determined up to the number $\hat{\theta}$. For any $0 \leq n \leq \mathcal{N}_{f}+s-1$, suppose that the phases $\left\{\theta\left(f\left(t_{0}\right)\right)\right\} \cup\left\{\theta\left(f\left(k+t_{k_{j}}\right)\right): k=\right.$ $1, \ldots, n-1, j=1,2,3\}$ have been determined (up to the global real number $\widehat{\theta}$ ) with probability 1 . Correspondingly, $\left\{c_{k}\right\}_{k=0}^{n-1}$ haven been determined (up to the scalar $e^{\mathbf{i} \widehat{\theta}}$ ) with probability 1 . Now by Lemma 2.12, Theorem 2.4 and Lemma 2.3, $\theta\left(f\left(n+t_{n_{1}}\right)+\widehat{\theta}\right.$ can be determined (up to the global real number $\theta$ ) via Approach II-B (2.31) with probability 1 . Then $c_{n}$ can be determined (up to the scalar $\left.e^{\mathbf{i} \widehat{\theta}}\right)$ with probability 1 . The proof can be concluded by the recursion.

\section{G. Proof of Proposition 2.6}

$\operatorname{By} \operatorname{supp}(\phi) \subseteq(0, s)$ we just need to prove that $\left\{c_{n}\right\}_{n=0}^{L-1}$ can be determined with probability 1 , up to a unimodular scalar. As in section II-F, with probability $1, c_{0}$ is determined by $\left|f\left(t_{0}\right)\right|$, up to a unimodular scalar $e^{\mathbf{i} \gamma}$. Suppose that with probability $1,\left\{c_{k}\right\}_{k=0}^{n-1}$ are determined up to $e^{\mathbf{i} \gamma}$. Then by the same argument as in section II-F we can prove that with the same probability, $c_{n}$ can be determined, up to $e^{\mathbf{i} \gamma}$, by $\left|f\left(n+t_{n_{i}}\right)\right|, i=1,2,3$. The proof is concluded.

H. Numerical simulation: random PLS of complex-valued and highly oscillatory chirps

This section is to verify Theorem 2.5. Our test SIS $V_{\mathrm{ca}}\left(\phi_{a, b, p}\right)$ is related with [34, section 6.3.1]. Specifically,

$$
\begin{aligned}
& \phi_{a, b, p}(x) \\
& =\frac{2}{3} \sqrt{2 \pi|b|} e^{-\mathbf{i} \frac{a(x-2)^{2}}{2 b}} e^{-\mathbf{i} \frac{p(x-2)}{b}} \cos ^{2} \frac{\pi(x-2)}{4} \chi_{(0,4)}(x) .
\end{aligned}
$$

By section I-C1, both $\phi_{4,0.8,1}$ and $\phi_{50,0.8,1}$ are GHCgenerators. The test signal

$$
f_{a}(x):=\sum_{n=0}^{15} c_{n} \phi_{a, 0.8,1}(x-n),
$$

where $a=4,50$ and $\mathcal{G}\left(f_{a}\right)<3$. See Fig. II.1 for their graphs. In Fig. II.2 we also plot the phase function $\theta\left(f_{a}(x)\right)$ defined via $f_{a}(x)=\left|f_{a}(x)\right| e^{\mathbf{i} \theta\left(f_{a}(x)\right)}$. Clearly, the two signals are highly oscillatory. By Theorem 2.5, $f_{a}(x)$ can be determined with probability 1 , up to a unimodular, by the random samples $\left\{\left|f_{a}\left(t_{0}\right)\right|\right\} \bigcup\left\{\mid f_{a}(n+\right.$ $\left.\left.t_{n_{1}}\right)|,| f_{a}\left(n+t_{n_{2}}\right)|,| f_{a}\left(n+t_{n_{3}}\right) \mid: n=1, \ldots, 18\right\}$, where $t_{0}, t_{n_{1}}, t_{n_{2}}, t_{n_{3}} \sim \mathbf{U}(0,1)$. In the noiseless setting, $10^{3}$ trials are conducted to determine $f_{a}(x)$ by Approach II-B. The error is defined as

$$
\begin{aligned}
& \operatorname{Error}\left(f_{a}\right) \\
& :=\log _{10}\left(\min _{\gamma \in(0,2 \pi]}\left\|\left\{c_{k}\right\}-e^{\mathbf{i} \gamma}\left\{\widetilde{c}_{k}\right\}\right\|_{2} /\left\|\left\{c_{k}\right\}\right\|_{2}\right),
\end{aligned}
$$

where $\left\{\widetilde{c}_{k}\right\}$ is the coefficient sequence of the reconstruction version $\widetilde{f}_{a}(x)=\sum_{n=0}^{15} \widetilde{c}_{n} \phi_{a, 0.8,1}(x-n)$. Approach II-B is considered to be successful if $\operatorname{Error}\left(f_{a}\right) \leq-1.8$. The cumulative distribution function $(\mathrm{CDF})$ of $\operatorname{Error}\left(f_{a}\right)$ is defined as

$$
\operatorname{CDF}(x)=\frac{\#\left(\operatorname{Error}\left(f_{a}\right) \leq x\right)}{10^{3}} .
$$

Fig. II. 3 confirms that with probability 1 , the signals can be determined in the noiseless setting.

In what follows we examine the robustness of Approach II-B to noise corruption. The observed values of $\left\{\left|f\left(t_{0}\right)\right|\right\} \cup\left\{\left|f\left(n+t_{n_{1}}\right)\right|,\left|f\left(n+t_{n_{2}}\right)\right|,\left|f\left(n+t_{n_{3}}\right)\right|: n=\right.$ 

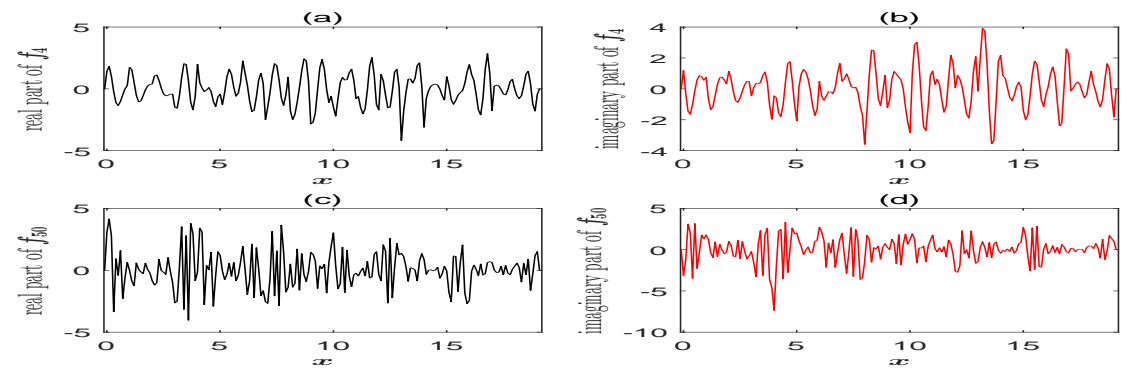

Fig. II.1: (a) The real part of $f_{4}$; (b) The imaginary part of $f_{4}$; (c) The real part of $f_{50}$; (d) The imaginary part of $f_{50}$.
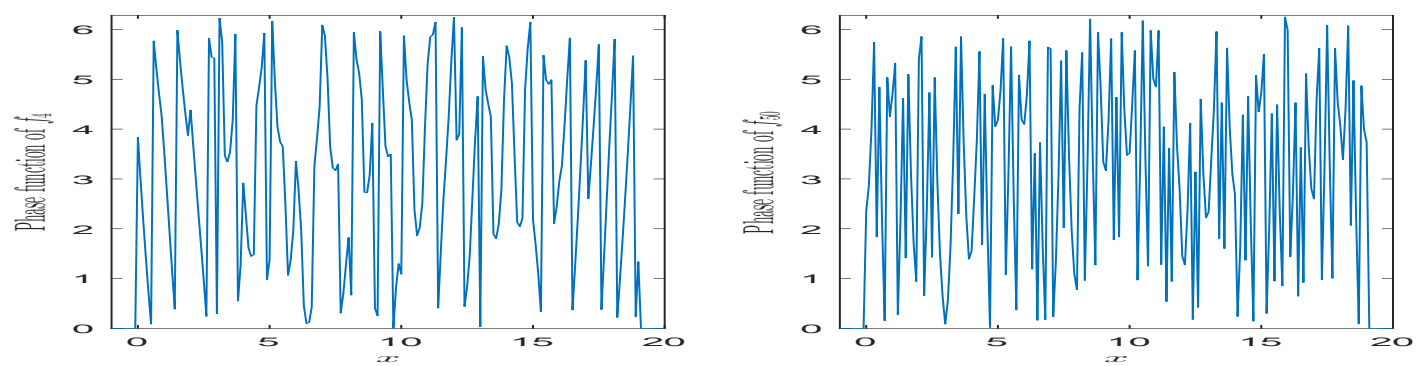

Fig. II.2: (a) The phase function $\theta\left(f_{4}(x)\right)$ of $f_{4}$; (b) The phase function $\theta\left(f_{50}(x)\right)$ of $f_{50}$.
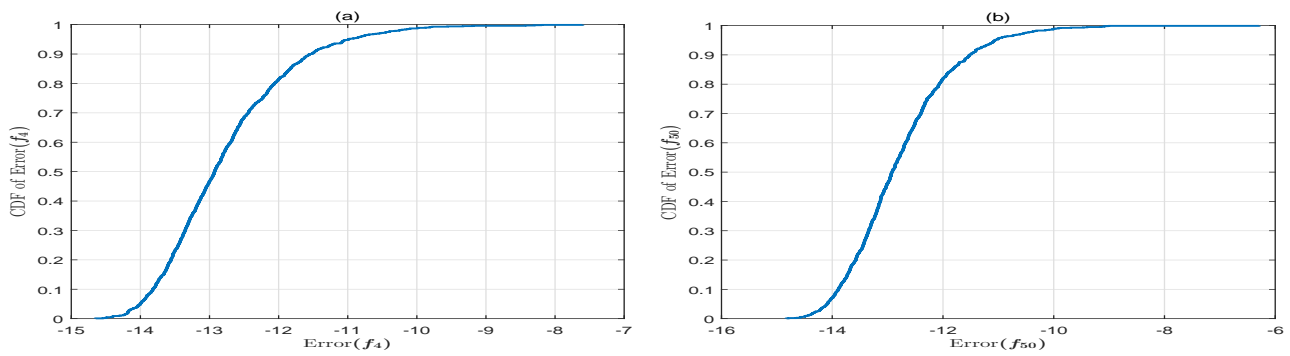

Fig. II.3: (a) The CDF of Error $\left(f_{4}\right)$ in the noiseless setting; (b) The CDF of Error $\left(f_{50}\right)$ in the noiseless setting.

\begin{tabular}{|c|c|c|c|c|c|c|c|c|c|}
\hline$f_{f_{a}} \quad$ SNR & 50 & 60 & 70 & 80 & 90 & 100 & 110 & 120 & 130 \\
\hline$f_{4}$ & 0.0070 & 0.0940 & 0.2800 & 0.4830 & 0.6440 & 0.7990 & 0.8860 & 0.9410 & 0.9970 \\
\hline$f_{50}$ & 0.0240 & 0.1770 & 0.4040 & 0.6270 & 0.7330 & 0.8430 & 0.9090 & 0.9520 & 0.9880 \\
\hline
\end{tabular}

TABLE II.1: Success rate vs noise level (SNR).

$1, \ldots, 18\}$ in a trial are denoted by $\left\{\left|f\left(\widehat{t}_{0}\right)\right|\right\} \cup\{\mid f(n+$ $\left.\left.\widehat{t}_{n_{1}}\right)|| f,\left(n+\widehat{t}_{n_{2}}\right)|| f,\left(n+\widehat{t}_{n_{3}}\right) \mid: n=1, \ldots, 18\right\}$. We add the Gaussian noise $\varepsilon \sim \mathbf{N}\left(0, \sigma^{2}\right)$ to the noiseless samples. That is, we employ the noisy samples $\left\{\left|f_{a}\left(\widehat{t}_{0}\right)\right|+\varepsilon\right\} \cup$ $\left\{\left|f_{a}\left(n+\widehat{t}_{n_{1}}\right)\right|+\varepsilon,\left|f_{a}\left(n+\widehat{t}_{n_{2}}\right)\right|+\varepsilon,\left|f_{a}\left(n+\widehat{t}_{n_{3}}\right)\right|+\varepsilon:\right.$ $n=1, \ldots, 18\}$ to conduct Approach II-B. The variance $\sigma^{2}$ is chosen such that the desired signal to noise ratio (SNR) is expressed by

$$
\mathrm{SNR}=10 \log _{10}\left(\frac{\left\|\mathbf{F}_{a}\right\|_{2}^{2}}{55 \sigma^{2}}\right),
$$

where $\left\|\mathbf{F}_{a}\right\|_{2}^{2}=\left|f_{a}\left(\widehat{t}_{0}\right)\right|^{2}+\sum_{k=1}^{3} \sum_{n=1}^{18}\left|f_{a}\left(n+\widehat{t}_{n_{k}}\right)\right|^{2}$. In the noisy setting, $10^{3}$ trials are also conducted to reconstruct $f_{4}(x)$ and $f_{50}(x)$, respectively. Their reconstruction success rates $(\mathrm{CDF}(-1.8))$ are recorded in Table II.1.

\section{RANDOM PHASELESS SAMPLING OF CAUSAL AND REAL-VALUED SIGNALS IN REAL-GENERATED SISS}

Throughout this section, let $\varphi$ be a real-valued GHCgenerator such that $\operatorname{supp}(\varphi) \subseteq(0, s)$ with the integer $s \geq$ 
2. This section focuses on the PLS of real-valued signals in

$$
V_{\mathrm{ca}}(\varphi)=\left\{\sum_{k=0}^{\infty} c_{k} \varphi(\cdot-k):\left\{c_{k} \in \mathbb{R}\right\} \in \ell^{2}, c_{0} \neq 0\right\} .
$$

Some denotations and definitions are helpful for discussion. Suppose that the signal $f \in V_{\mathrm{ca}}(\varphi)$ is denoted by

$$
f=\sum_{k=0}^{\infty} c_{k} \varphi(\cdot-k) .
$$

As in section II-B denote $\mathcal{N}_{f}=\sup \left\{k: c_{k} \neq 0\right\}$. As in (2.12), define the index set $I_{n}$ by

$$
I_{n}= \begin{cases}\{0,1, \ldots, n-1\}, & 1 \leq n \leq s-1 \\ \{n-s+1, \ldots, n-1\}, & n \geq s\end{cases}
$$

For $n \geq 1$ and the signal $f$ in (3.35), define an auxiliary function

$$
v_{n, f}^{\Re}(x):=\sum_{k \in I_{n}} c_{k} \varphi(n+x-k), x \in(0,1) .
$$

Moreover, define

$$
\begin{aligned}
A_{n, f}^{\Re}(x, y):=\frac{|f|(n+x)}{|\varphi|^{2}(x)}[ & \varphi(x) \varphi(y) v_{n, f}^{\Re}(y) \\
& \left.-v_{n, f}^{\Re}(x) \varphi^{2}(y)\right],
\end{aligned}
$$

and

$$
\begin{aligned}
C_{n, f}^{\Re}(x, y) & :=|f|^{2}(n+y)-\left|v_{n, f}^{\Re}\right|^{2}(y) \\
& +\frac{2 v_{n, f}^{\Re}(x) v_{n, f}^{\Re}(y) \varphi(y)}{\varphi(x)} \\
& -\frac{|\varphi|^{2}(y)}{|\varphi|^{2}(x)}\left[|f|^{2}(n+x)+\left|v_{n, f}^{\Re}\right|^{2}(x)\right],
\end{aligned}
$$

whenever $x, y \in(0,1)$ such that $\varphi(x) \neq 0$. The maximum gap $\mathcal{G}_{f}$ is defined via Definition 2.8 with $\phi$ replaced by $\varphi$. The sign function $\operatorname{sgn}(x)$ takes $1,-1$ and 0 when $x>$ $0, x<0$ and $x=0$, respectively.

\section{A. Random PLS of real-valued signals in $V_{c a}(\varphi)$}

We next establish the main theorem of this section. It is the counterpart of Theorem 2.5.

Theorem 3.1: Let $\varphi$ be a real-valued GHC-generator such that $\operatorname{supp}(\varphi) \subseteq(0, s)$ with the integer $s \geq 2$. Then any nonseparable and real-valued signal $f \in V_{\mathrm{ca}}(\varphi)$ can be determined (up to a sign) with probability 1 by the random samples $\left\{\left|f\left(t_{0}\right)\right|\right\} \cup\left\{\left|f\left(n+t_{n_{1}}\right)\right|,\left|f\left(n+t_{n_{2}}\right)\right|\right.$ : $n=1, \ldots, \infty\}$, where the i.i.d random variables $\left\{t_{0}\right\} \cup$ $\left\{t_{n_{1}}, t_{n_{2}}: n=1, \ldots, \infty\right\} \sim \mathbf{U}(0,1)$.

Proof: Since $f$ is nonseparable, by the same argument as in Lemma 2.9 we have $\mathcal{G}_{f}<s-1$, which together with $\varphi$ being a real-valued GHC-generator leads to the probability $P\left(\left|f\left(k+t_{k_{i}}\right)\right|>0\right)=1$ for any $k \in\left\{0, \ldots, \mathcal{N}_{f}+s-1\right\}$. Clearly, $P\left(\left|\varphi\left(t_{n_{i}}\right)\right|>0\right)=1$. As in section II-F, it is sufficient to prove that the phases $\left\{\theta\left(f\left(t_{0}\right)\right)\right\} \cup\{\theta(f(n+$ $\left.\left.\left.t_{n_{i}}\right)\right): n=1, \ldots, \mathcal{N}_{f}+s-1, i=1,2\right\}$ can be determined, up to the constant $\pi$, with probability 1 .
Denote $f=\sum_{k=0}^{\infty} c_{k} \varphi(\cdot-k)$. By the similar argument as in the proof of Lemma 2.10, we can prove that

$$
P\left(A_{n, f}^{\Re}\left(t_{n_{1}}, t_{n_{2}}\right) \neq 0\right)=1, n=1, \ldots, \mathcal{N}_{f}+s-1 .
$$

Assume that $z_{0}=e^{\mathbf{i} \theta\left(f\left(t_{0}\right)\right)} \in\{1,-1\}$ is assigned exactly. As previously, $P\left(\left|f\left(n+t_{n_{i}}\right)\right|>0\right)=P\left(\left|\varphi\left(t_{n_{i}}\right)\right|>0\right)=$ 1. Then

$$
f\left(t_{0}\right)=z_{0}\left|f\left(t_{0}\right)\right|,
$$

and $c_{0}=\frac{z_{0}\left|f\left(t_{0}\right)\right|}{\varphi\left(t_{0}\right)}$ with probability 1 . We next determine $\theta\left(f\left(t_{1_{1}}+1\right)\right)$ and $c_{1}$. Similarly to (2.24), we have

$$
\left\{\begin{aligned}
\left|v_{1, f}^{\Re}\left(t_{1_{1}}\right)+c_{1} \varphi\left(t_{1_{1}}\right)\right| & =\left|f\left(1+t_{1_{1}}\right)\right|, \\
\left|v_{1, f}^{\Re}\left(t_{1_{2}}\right)+c_{1} \varphi\left(t_{1_{2}}\right)\right| & =\left|f\left(1+t_{1_{2}}\right)\right| .
\end{aligned}\right.
$$

Denote $f\left(1+t_{1_{1}}\right):=z_{1}\left|f\left(1+t_{1_{1}}\right)\right|$ with $z_{1} \in\{1,-1\}$ to be determined. By the similar argument as in (2.27), we can prove that $z_{1}$ is the solution to

$$
A_{1, f}^{\Re}\left(t_{1_{1}}, t_{1_{2}}\right) z^{2}-C_{1, f}^{\Re}\left(t_{1_{1}}, t_{1_{2}}\right) z+A_{1, f}^{\Re}\left(t_{1_{1}}, t_{1_{2}}\right)=0 .
$$

It follows from (3.39) that with probability 1 , there exist at most two solutions to the above equation. Note that the product of the two solutions is 1 . Then there exists a unique solution with the same probability. More precisely,

$$
z_{1}=\operatorname{sgn}\left(\frac{C_{1, f}^{\Re}\left(t_{1_{1}}, t_{1_{2}}\right)}{A_{1, f}^{\Re,}\left(t_{1_{1}}, t_{1_{2}}\right)}\right) .
$$

Therefore under the assumption (3.40), $c_{1}=$ $\frac{z_{1}\left|f\left(1+t_{1_{1}}\right)\right|-v_{1, f}\left(t_{1_{1}}\right)}{\varphi\left(t_{1_{1}}\right)}$ with probability 1 . And $\theta\left(f\left(1+t_{1_{i}}\right)\right)$ can be determined with probability 1 . Continuing the above procedures, $\left\{\theta\left(f\left(t_{0}\right)\right)\right\} \cup\left\{\theta\left(f\left(n+t_{n_{i}}\right)\right): n=\right.$ $\left.1, \ldots, \mathcal{N}_{f}+s-1, i=1,2\right\}$ can be determined with the same probability.

Contrary to (3.40), we next assign

$$
f\left(t_{0}\right)=-z_{0}\left|f\left(t_{0}\right)\right| \text {. }
$$

Under (3.44), we shall prove that $\left\{\theta\left(f\left(t_{0}\right)\right)+\pi\right\} \cup$ $\left\{\theta\left(f\left(n+t_{n_{i}}\right)\right)+\pi: n=1, \ldots, \mathcal{N}_{f}+s-1, i=1,2\right\}$ or $\widetilde{f}=\sum_{k=0}^{\infty} \widetilde{c}_{k} \varphi(\cdot-k)$ can be determined with probability 1 , where $\widetilde{c}_{k}=-c_{k}$. First it follows from $P\left(\left|\varphi\left(t_{0}\right)\right| \neq\right.$ $0)=1$ that

$$
\widetilde{c}_{0}=-\frac{z_{0}\left|f\left(t_{0}\right)\right|}{\varphi\left(t_{0}\right)}=-c_{0} .
$$

Then $\theta\left(\tilde{f}\left(t_{0_{i}}\right)\right)=\theta\left(f\left(t_{0_{i}}\right)\right)+\pi$. By (3.37), (3.38) and (3.45), we have $A_{1, \tilde{f}}^{\Re}\left(t_{1_{1}}, t_{1_{2}}\right)=-A_{1, f}^{\Re}\left(t_{1_{1}}, t_{1_{2}}\right)$ and $C_{1, \tilde{f}}^{\Re}\left(t_{1_{1}}, t_{1_{2}}\right)=C_{1, f}^{\Re}\left(t_{1_{1}}, t_{1_{2}}\right)$. Moreover, as in (3.42), $\operatorname{sgn}\left(\tilde{f}\left(1+t_{1_{1}}\right)\right)$ is the solution to

$$
A_{1, \widetilde{f}}^{\Re}\left(t_{1_{1}}, t_{1_{2}}\right) z^{2}-C_{1, \widetilde{f}}^{\Re}\left(t_{1_{1}}, t_{1_{2}}\right) z+A_{1, \widetilde{f}}^{\Re}\left(t_{1_{1}}, t_{1_{2}}\right)=0 .
$$



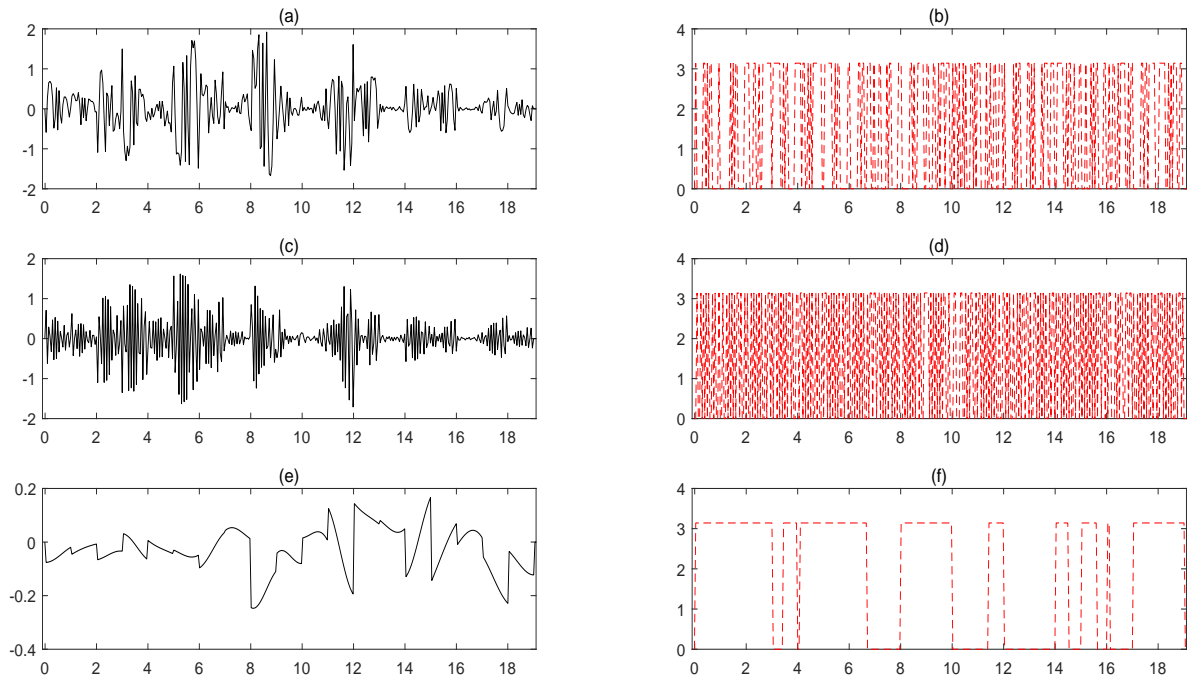

Fig. III.4: (a) The graph of $f_{10,-0.2381,1}$; (b) The graph of the phase function of $f_{10,-0.2381,1}$; (c) The graph of $f_{50,-0.2381,1}$; (d) The graph of the phase function of $f_{50,-0.2381,1}$; (e) The graph of $f_{10^{-6},-0.0038,0}$; (f) The graph of the phase function of $f_{10^{-6},-0.0038,0}$.
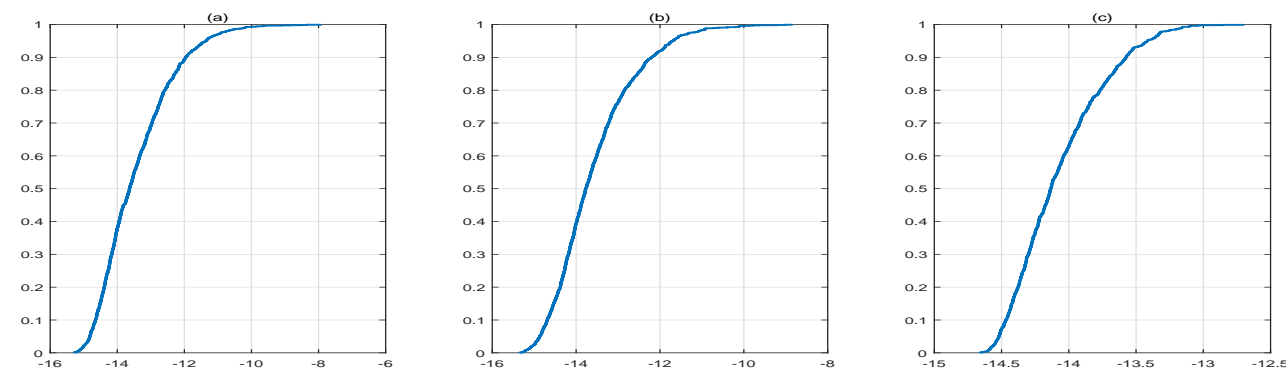

Fig. III.5: (a) The CDF of Error $\left(f_{10,-0.2381,1}\right)$ in the noiseless setting; (b) The CDF of Error $\left(f_{50,-0.2381,1}\right)$ in the noiseless setting; (c) The CDF of Error $\left(f_{10^{-6},-0.0038,0}\right)$ in the noiseless setting
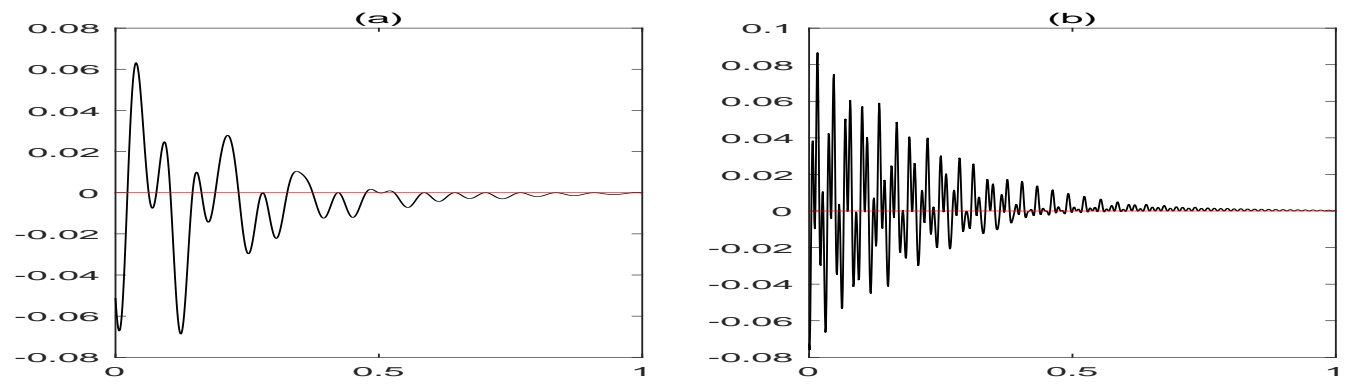

Fig. III.6: (a) The graph of $A_{1, g_{10}}^{\Re}(0.5, x)$; (b) The graph of $A_{1, g_{50}}^{\Re}(0.5, x)$ 
As in (3.43), the solution is given by

$$
z=\operatorname{sgn}\left(\frac{C_{1, \tilde{f}}^{\Re}\left(t_{1_{1}}, t_{1_{2}}\right)}{A_{1, \tilde{f}}^{\Re}\left(t_{1_{1}}, t_{1_{2}}\right)}\right)=-\operatorname{sgn}\left(\frac{C_{1, f}^{\Re}\left(t_{1_{1}}, t_{1_{2}}\right)}{A_{1, f}^{\Re}\left(t_{1_{1}}, t_{1_{2}}\right)}\right) .
$$

Then $\theta\left(\widetilde{f}\left(1+t_{1_{1}}\right)\right)=\theta\left(f\left(1+t_{1_{1}}\right)\right)+\pi$. Consequently, $\widetilde{c}_{1}=-c_{1}$ and $\theta\left(\widetilde{f}\left(1+t_{1_{2}}\right)\right)=\theta\left(f\left(1+t_{1_{2}}\right)\right)+\pi$. By recursion on $n$, we can prove that $\left\{\theta\left(f\left(t_{0}\right)\right)+\pi\right\} \cup$ $\left\{\theta\left(f\left(n+t_{n_{i}}\right)\right)+\pi: n=1, \ldots, \mathcal{N}_{f}+s-1, i=1,2\right\}$ can be determined with probability 1 .

The following proposition concerns on the local reconstruction. It is the counterpart of Proposition 2.6.

Proposition 3.2: Let $\varphi$ and $f$ be as in Theorem 3.1. Then for any integer $L>1$, the restriction $f_{[0, L]}$ of $f$ on $[0, L]$ can be determined with probability 1 , up to a sign, by the random samples $\left\{\left|f\left(t_{0}\right)\right|\right\} \cup\left\{\left|f\left(n+t_{n_{1}}\right)\right|, \mid f(n+\right.$ $\left.\left.t_{n_{2}}\right) \mid: n=1, \ldots, L-1\right\}$, where the i.i.d random variables $\left\{t_{0}\right\} \cup\left\{t_{n_{1}}, t_{n_{2}}: n=1, \ldots, L-1\right\} \sim \mathbf{U}(0,1)$.

Proof: The proof is based on that of Theorem 3.1. And it can be concluded by the similar argument as in section II-G.

\section{B. PD-CR for nonseparable real-valued signals in $V_{c a}(\varphi)$}

Let $\left\{\widehat{t}_{0}\right\} \cup\left\{\widehat{t}_{n_{1}}, \widehat{t}_{n_{2}}: n=1, \ldots, \infty\right\}$ be the observed values of random variables $\left\{t_{0}\right\} \cup\left\{t_{n_{1}}, t_{n_{2}}: n=\right.$ $1, \ldots, \infty\}$ in Theorem 3.1. Based on the proof of Theorem 3.1 , in what follows we establish an approach for the PLS of nonseparable real-valued signals in $V_{\mathrm{ca}}(\varphi)$.

\section{Approach III-B}

Input: Samples $\left\{\left|f\left(\widehat{t}_{0}\right)\right|\right\} \cup\left\{\left|f\left(k+\widehat{t}_{k_{j}}\right)\right|: j=1,2, k=\right.$ $1, \ldots, n\}$ where $\widehat{t}_{0}, \widehat{t}_{k_{j}} \in(0,1)$ and $n \leq \mathcal{N}_{f}+s-1$. Assign initial phase $\theta\left(f\left(\widehat{t}_{0}\right)\right)=\widetilde{\theta}_{0} \in\{0, \pi\} ; c_{0}=$ $e^{\mathbf{i} \widetilde{\theta}_{0}}\left|f\left(\widehat{t}_{0}\right)\right| / \varphi\left(\widehat{t}_{0}\right)$.

Output: $\left\{c_{k}\right\}_{k=0}^{n}$ and $\left\{\theta\left(f\left(\widehat{t}_{0}\right)\right)\right\} \cup\left\{\theta\left(f\left(k+\widehat{t}_{k_{j}}\right)\right): j=\right.$ $1,2, k=1, \ldots, n\}$.

Recursion assumption: Assume that the phases $\left\{\theta\left(f\left(k+\widehat{t}_{k_{j}}\right)\right): j=1,2, k=1, \ldots, n-1\right\}$ and coefficients $\left\{c_{k}\right\}_{k=0}^{n-1}$ have been recovered. Then $\{\theta(f(n+$ $\left.\left.\left.\widehat{t}_{n_{j}}\right)\right): j=1,2\right\}$ and $c_{n}$ are recovered by the following steps:

step 1: Compute $v_{n, f}^{\Re}\left(\widehat{t}_{n_{1}}\right), \quad A_{n, f}^{\Re}\left(\widehat{t}_{n_{1}}, \widehat{t}_{n_{2}}\right)$ and $C_{n, f}^{\Re}\left(\widehat{t}_{n_{1}}, \widehat{t}_{n_{2}}\right)$ by (3.36), (3.37) and (3.38), respectively.

step 2: $\theta\left(f\left(n+\widehat{t}_{n_{1}}\right)\right) \in\{0, \pi\}$ is recovered by computing $e^{\mathrm{i} \theta\left(f\left(n+\widehat{t}_{n_{1}}\right)\right)}=\operatorname{sgn}\left(\frac{C_{n, f}^{\Re}\left(\widehat{t}_{n_{1}}, \widehat{t}_{n_{2}}\right)}{A_{n, f}^{\Re}\left(\widehat{t}_{n_{1}}, \widehat{t}_{n_{2}}\right)}\right)$. And $c_{n}=$

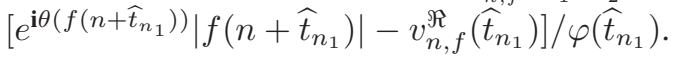

C. Numerical simulation: costing small number of samples to reconstruct highly oscillatory real-valued chirps

This section is to examine the efficiency of Approach

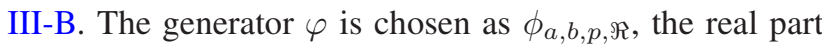
of $\phi_{a, b, p}$ defined in section II-H. The test signal is

$$
f_{a, b, p}(t)=\sum_{n=0}^{15} c_{n, a} \phi_{a, b, p, \Re}(t-n),
$$

where $c_{0, a} \neq 0, c_{n, a} \in \mathbb{R}$. It is easy to check that $f_{a, b, p}(t)$ can be rewritten as the real-valued chirp form (c.f. [40]): $A(t) \cos (\lambda v(t))$ with $A(\cdot) \geq 0$. By the analysis in section I-C2, we can check that $\phi_{10,-0.238,1, \Re,} \phi_{50,-0.238,1, \Re}$ and

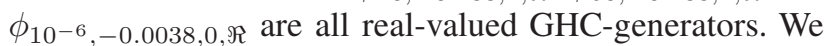
choose $f_{10,-0.2381,1}, f_{50,-0.2381,1}$ and $f_{10^{-6,-0.0038,0}}$ as test signals. Their graphs are plotted in Fig. III.4 (a, c, e). Moreover, the phase function $\theta\left(f_{a, b, p}(x)\right)$, taking 0 and $\pi$ when $f_{a, b, p}(x) \geq 0$ and $f_{a, b, p}(x)<0$, respectively, is plotted in Fig. III.4 (b, d, f).

Fig. III.4 (b, d, f) imply that $f_{10,-0.2381,1}$ and $f_{50,-0.2381,1}$ are much more oscillatory than $f_{10^{-6},-0.0038,0}$. It should be noted that a great number of deterministic samples are necessary for the local reconstructions of $f_{10,-0.2381,1}$ and $f_{50,-0.2381,1}$. To make this point, define

$$
g_{a}(t)=\sum_{n=0}^{1} c_{n, a} \phi_{a,-0.238,1, \Re}(t-n), t \in(0,2),
$$

where $c_{0,10}=0.7064, c_{1,10}=-0.6183, c_{0,50}=-0.5874$ and $c_{1,50}=0.2659$ are as in (3.46). Clearly,

$$
g_{a}(t)=f_{a,-0.2381,1}(t), t \in(0,2),
$$

and the reconstruction of $g_{a}$ is equivalent to ones of $c_{0, a}$ and $c_{1, a}$. Suppose that $c_{0, a}$ and $c_{1, a}$ can be recovered by any $\stackrel{L}{L}$ deterministic samples $\left\{\left|g_{a}\left(\widehat{t}_{0}\right)\right|,\left|g_{a}\left(1+\widehat{t}_{1_{i}}\right)\right|: i=\right.$ $1, \ldots, \stackrel{\circ}{L}-1\}$, where $\widehat{t}_{0}, \widehat{t}_{1_{i}} \in(0,1)$. We next estimate $\stackrel{\circ}{L}$. It

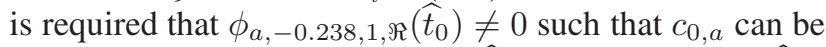
determined, up to a sign, by $\left|g_{a}\left(\widehat{t}_{0}\right)\right|$. Otherwise, $\left|g_{a}\left(\widehat{t}_{0}\right)\right|$ is useless for determining $c_{0, a}$. Without loss of generality, assume that $c_{0, a}$ is determined and $\left|g_{a}\left(1+\widehat{t}_{1_{i}}\right)\right| \neq 0$. Next we need to determine $c_{1, a}$. Then the determination of $c_{1, a}$ is equivalent to the determination of $z:=\operatorname{sgn}\left(g_{a}\left(1+\widehat{t}_{1_{1}}\right)\right)$. By the analysis in (3.41), $z$ is the solution to (3.42) with $A_{1, f}^{\Re}\left(t_{1_{1}}, t_{1_{2}}\right)$ and $C_{1, f}^{\Re}\left(t_{1_{1}}, t_{1_{2}}\right)$ therein replaced by $A_{1, g_{a}}^{\Re}\left(\widehat{t}_{1_{1}}, \widehat{t}_{1_{j}}\right)$ and $C_{1, g_{a}}^{\Re}\left(\widehat{t}_{1_{1}}, \widehat{t}_{1_{j}}\right)$, respectively, where $j \neq 1$. Clearly, $z$ can be determined if and only if $A_{1, g_{a}}^{\Re}\left(\widehat{t}_{1_{1}}, \widehat{t}_{1_{j}}\right) \neq 0$. As an example, we choose $\widehat{t}_{1_{1}}=0.5$ without bias. See the graph of $A_{1, g_{a}}^{\Re}(0.5, x)$ on $(0,1)$ in Fig. III.6. Obviously the number of zeros of $A_{1, g_{a}}^{\Re}(0.5, x)$ on $(0,1)$ is much larger than 2 . Especially, we found that the number of zeros of $A_{1, g_{50}}^{\Re}(0.5, x)$ is not smaller than 256. Then we need at least 257 additional deterministic samples on $(1,2)$ to avoid $A_{1, g_{50}}^{\Re}(0.5, x)=0$. Therefore 


\begin{tabular}{|c|cccccccc}
\hline SNR & 35 & 40 & 50 & 60 & 70 & 80 & 90 & 100 \\
\hline$f_{a, b, p}$ & & & & & & & \\
\hline$f_{50,-0.2381,1}$ & 0.0100 & 0.0590 & 0.2460 & 0.5290 & 0.6870 & 0.8450 & 0.9200 & 0.9620 \\
$f_{10,-0.2381,1}$ & 0.0160 & 0.0470 & 0.2520 & 0.4590 & 0.6570 & 0.8540 & 0.9090 & 0.9560 \\
$f_{10}-6,-0.0038,0$ & 0.1100 & 0.3370 & 0.7390 & 0.9080 & 0.9520 & 0.9800 & 0.9850 & 0.9960 \\
\hline
\end{tabular}

TABLE III.2: Success rate vs noise level (SNR).

for reconstructing $g_{50}, \stackrel{\circ}{L} \geq \mathbf{2 5 9}$ although it is determined by only two coefficients. By Proposition 3.2, however, $g_{a}$ can be determined, with probability 1 , by just three random samples. Allowing for (3.48) we just need to check the recovery efficiency of $f_{a,-0.2381,1}$.

In the present simulation, by the random samples

$$
\begin{aligned}
& \left\{\left|f_{a, b, p}\left(t_{0}\right)\right|\right\} \cup\left\{\left|f_{a, b, p}\left(n+t_{n_{1}}\right)\right|,\left|f_{a, b, p}\left(n+t_{n_{2}}\right)\right|:\right. \\
& \quad n=1, \ldots, 18\},
\end{aligned}
$$

$10^{3}$ trials of Approach III-B are conducted to recover $f_{a, b, p}$, where $\left\{t_{0}\right\} \cup\left\{t_{n_{1}}, t_{n_{2}}: n=1, \ldots, 18\right\} \sim \mathbf{U}(0,1)$. The recovery error is defined as

$$
\begin{array}{r}
\operatorname{Error}\left(f_{a, b, p}\right):=\log _{10}\left(\min _{\gamma \in\{1,-1\}} \|\left\{c_{k, a}\right\}\right. \\
\left.-\gamma\left\{\widetilde{c}_{k, a}\right\}\left\|_{2} /\right\|\left\{c_{k, a}\right\} \|_{2}\right),
\end{array}
$$

where $\left\{\widetilde{c}_{k, a}\right\}$ is the recovery version of $\left\{c_{k, a}\right\}$. As in section II-H, the approach is considered successful if $\operatorname{Error}\left(f_{a, b, p}\right) \leq-1.8$, and the cumulative distribution function (CDF) of the error is defined via (2.33). Clearly, Fig. III.5 implies that $f_{10,-0.2381,1}, f_{50,-0.2381,1}$ and $f_{10^{-6},-0.0038,0}$ can be recovered perfectly in the noiseless setting. To check the stability to noise, we also conduct $10^{3}$ trials in the noisy setting. As in section II-H, we add the Gaussian noise $\varepsilon \sim \mathbf{N}\left(0, \sigma^{2}\right)$ to the observed noiseless samples in (3.49). The variance $\sigma^{2}$ is chosen via (2.34) with 55 therein replaced by 37 such that the desired SNR can be expressed. As in the noiseless case, $10^{3}$ trials are also conducted. The success rates $(\mathrm{CDF}(-1.8))$ are recorded in Table III.2.

Comparing Table II.1 and Table III.2 we found that, for the low SNR (e.g. $\leq 60)$, the stability to noise in the present simulation (real-valued case) is much stronger than that in section III-C (complex-valued case). We next interpret this from the phase distribution perspective.

Remark 3.3: For a real-valued signal $f \in V(\varphi)$, its phase function $\theta(f(x))$ has only two values: 0 and $\pi$. Since the samples in (3.49) are perturbed, unavoidably so

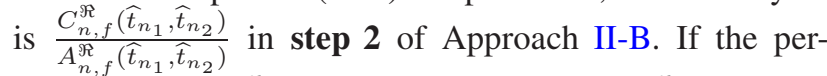
turbation $\epsilon$ of $\frac{C_{n, f}^{\Re}\left(\widehat{t}_{n_{1}}, \widehat{t}_{n_{2}}\right)}{A_{n, f}^{\Re}\left(\hat{t}_{n_{1}}, t_{n_{2}}\right)}$ satisfies $|\epsilon|<\left|\frac{C_{n, f}^{\Re}\left(\widehat{t}_{n_{1}}, \widehat{t}_{n_{2}}\right)}{A_{n, f}^{\Re}\left(\bar{t}_{n_{1}}, \hat{t}_{n_{2}}\right)}\right|$, then $\theta\left(f\left(n+\widehat{t}_{n_{1}}\right)\right)$ can be decoded exactly through step 2 . Unlike the real-valued case, Fig. II.2 implies that the phases of the complex-valued signals in section II-H are much more complicated. Therefore, it is no wonder that the stability in the present simulation is much stronger than that in section II-H.

On the other hand, it follows from Fig. III.4 (b, d, f) that the phase function of $f_{10^{-6},-0.0038,0}$ varies much more slowly than those of $f_{10,-0.2381,1}$ and $f_{50,-0.2381,1}$. And when SNR $(\leq 50)$ is low, numerical results in Table III.2 imply the much stronger stability for $f_{10^{-6},-0.0038,0}$.

Recall that the distribution and oscillation of the phase is the intrinsic property of a signal. Overall, the simulation results in section II-H and in the present section imply that the recovery stability to noise is related with the property.

\section{CONCLUSION}

We prove that the full spark property is not sufficient for the phaseless sampling in complex-generated shiftinvariant spaces (SISs) (Theorem 1.1). We establish a condition for decoding the phases of the samples (Theorem 2.4). Based on Theorem 2.4, we establish a reconstruction scheme in Approach II-B. Based on Approach II-B and the generalized Haar condition (GHC), nonseparable and causal (NC) signals in the complex-generated SISs can be determined with probability 1 if the random sampling density (SD) is not smaller than 3 (Theorem 2.5). Approach II-B is modified to Approach III-B such that it is more adaptive to real-valued $\mathrm{NC}$ signals in real-generated SISs. Based on Approach III-B and GHC, real-valued NC signals in the real-generated SISs can be determined with probability 1 if the random SD is not smaller than 2 (Theorem 3.1). Propositions 2.6 and 3.2 imply that the highly oscillatory signals can be determined locally, with probability 1 , by a very small number of random samples.

\section{APPENDiX}

\section{A. Proof of Lemma 2.10}

Since $t_{n_{1}}, t_{n_{2}}$ and $t_{n_{3}}$ are i.i.d random variables, we just need to prove $P\left(A_{n, f}\left(t_{n_{1}}, t_{n_{2}}\right)+B_{n, f}\left(t_{n_{1}}, t_{n_{2}}\right) \mathbf{i} \neq 0\right)=$ 1.

Define an event $\widetilde{\mathfrak{E}}_{n, 0}:=\left\{\phi\left(t_{n_{1}}\right) \bar{f}\left(n+t_{n_{1}}\right) \neq 0\right\}$ w.r.t $t_{n_{1}}$. By (2.14), we have

$\widetilde{\mathfrak{E}}_{n, 0}$

$=\left\{\phi\left(t_{n_{1}}\right)\left(\bar{v}_{n, f}\left(t_{n_{1}}\right)+\bar{c}_{n} \bar{\phi}\left(t_{n_{1}}\right)\right) \neq 0\right\}$

$=\left\{\sum_{k \in I_{n}} \bar{c}_{k} \phi\left(t_{n_{1}}\right) \bar{\phi}\left(n+t_{n_{1}}-k\right)+\bar{c}_{n}|\phi|^{2}\left(t_{n_{1}}\right) \neq 0\right\}$. 
First, it is easy to derive from Lemma 2.9 and the definition of $\mathcal{N}_{f}$ that, for every $n \in\left\{1,2, \ldots, \mathcal{N}_{f}+s-1\right\}$ there exists a nonzero coefficient in $\left\{c_{k}: k \in I_{n}\right\}$. Moreover, $\Lambda_{\phi, 2}$ in Proposition 2.1 satisfies GHC. Then

$$
\begin{aligned}
& \mu\left(\left\{t \in(0,1): \sum_{k \in I_{n}} \bar{c}_{k} \phi(t) \bar{\phi}(n+t-k)\right.\right. \\
& =0 .
\end{aligned}
$$

Therefore $P\left(\widetilde{\mathfrak{E}}_{n, 0}\right)=1$. Consequently, $P\left(\mathfrak{E}_{n, 0}\right)=1$ where $\mathfrak{E}_{n, 0}=\left\{\frac{\left|f\left(n+t_{n_{1}}\right)\right|}{|\phi|^{2}\left(t_{n_{1}}\right)} \neq 0\right\}$. Define an auxiliary (random) function w.r.t $t_{n_{1}}$ and $t_{n_{2}}$ by

$$
\begin{aligned}
& a_{n, f}\left(t_{n_{1}}, t_{n_{2}}\right)+b_{n, f}\left(t_{n_{1}}, t_{n_{2}}\right) \mathbf{i} \\
& :=\bar{\phi}\left(t_{n_{1}}\right) \phi\left(t_{n_{2}}\right) \bar{v}_{n, f}\left(t_{n_{2}}\right)-\bar{v}_{n, f}\left(t_{n_{1}}\right)|\phi|^{2}\left(t_{n_{2}}\right) .
\end{aligned}
$$

Direct observation on (2.15) leads to that

$$
\begin{aligned}
& A_{n_{f}(}\left(t_{n_{1}}, t_{n_{2}}\right)+B_{n, f}\left(t_{n_{1}}, t_{n_{2}}\right) \mathbf{i} \\
& =\frac{|f|\left(n+t_{n_{1}}\right)}{|\phi|^{2}\left(t_{n_{1}}\right)}\left(a_{n, f}\left(t_{n_{1}}, t_{n_{2}}\right)+b_{n, f}\left(t_{n_{1}}, t_{n_{2}}\right) \mathbf{i}\right) .
\end{aligned}
$$

As previously for every $n \in\left\{1,2, \ldots, \mathcal{N}_{f}\right\}$, there exists a nonzero coefficient in $\left\{c_{k}: k \in I_{n}\right\}$. Then by (2.13) we have $\bar{v}_{n, f} \not \equiv 0$. Now it follows from $\Lambda_{\phi, 2}$ in Proposition 2.1 satisfying GHC that $\phi \bar{v}_{n, f}$ and $|\phi|^{2}$ are linearly independent, which together with $P\left(\mathfrak{E}_{n, 0}\right)=1$ leads to $a_{n, f}(\cdot, \cdot)+b_{n, f}(\cdot, \cdot) \mathbf{i} \not \equiv 0$. Then

$$
\begin{aligned}
1 & \geq P\left(a_{n, f}\left(t_{n_{1}}, t_{n_{2}}\right)+b_{n, f}\left(t_{n_{1}}, t_{n_{2}}\right) \mathbf{i} \neq 0\right) \\
& \geq P\left(a_{n, f}\left(t_{n_{1}}, t_{n_{2}}\right)+b_{n, f}\left(t_{n_{1}}, t_{n_{2}}\right) \mathbf{i} \neq 0 \mid \mathfrak{E}_{n, 0}\right) P\left(\mathfrak{E}_{n, 0}\right) \\
& =P\left(a_{n, f}\left(t_{n_{1}}, t_{n_{2}}\right)+b_{n, f}\left(t_{n_{1}}, t_{n_{2}}\right) \mathbf{i} \neq 0 \mid \mathfrak{E}_{n, 0}\right) \\
& =1
\end{aligned}
$$

where $\Lambda_{\phi, 2}$ satisfying GHC is used again in the last identity. The proof is concluded.

\section{B. Proof of Lemma 2.11}

If $0<\frac{|f|\left(n+t_{n_{1}}\right)}{|\phi|^{2}\left(t_{n_{1}}\right)}<\infty$, then it follows from (5.53) that $\theta\left[A_{n, f}\left(t_{n_{1}}, t_{n_{2}}\right)+B_{n, f}\left(t_{n_{1}}, t_{n_{2}}\right) \mathbf{i}\right]=\theta\left[a_{n, f}\left(t_{n_{1}}, t_{n_{2}}\right)+\right.$ $\left.b_{n, f}\left(t_{n_{1}}, t_{n_{2}}\right) \mathbf{i}\right]$, where $a_{n, f}\left(t_{n_{1}}, t_{n_{2}}\right)+b_{n, f}\left(t_{n_{1}}, t_{n_{2}}\right) \mathbf{i}$ is defined in (5.52). By direct calculation, for $y \in(0,1)$ we have

$$
\begin{aligned}
& \Re\left(a_{n, f}\left(t_{n_{1}}, y\right)+\mathbf{i} b_{n, f}\left(t_{n_{1}}, y\right)\right) \\
& =a_{n, f}\left(t_{n_{1}}, y\right) \\
& =u_{t_{n_{1}}, f}\left(\phi_{\Re}^{2}(y)+\phi_{\Im}^{2}(y)\right) \\
& \quad+\sum_{k \in I_{n}}\left[\widetilde{c}_{t_{n_{1}}, k, \Re},\left(\phi_{\Re}(y) \phi_{\Re}(y+n-k)\right.\right. \\
& \left.\left.\quad+\phi_{\Im}(y) \phi_{\Im}(y+n-k)\right)\right] \\
& \quad-\sum_{k \in I_{n}}\left[\widetilde{c}_{t_{n_{1}}, k, \Im},\left(\phi_{\Im}(y) \phi_{\Re}(y+n-k)\right.\right. \\
& \left.\left.\quad-\phi_{\Re}(y) \phi_{\Im}(y+n-k)\right)\right],
\end{aligned}
$$

and

$$
\begin{aligned}
& \Im\left(a_{n, f}\left(t_{n_{1}}, y\right)+\mathbf{i} b_{n, f}\left(t_{n_{1}}, y\right)\right) \\
& \left.=b_{n, f}\left(t_{n_{1}}, y\right)\right) \\
& =v_{t_{n_{1}}, f}\left(\phi_{\Re}^{2}(y)+\phi_{\Im}^{2}(y)\right) \\
& +\sum_{k \in I_{n}}\left[\widetilde { c } _ { t _ { n _ { 1 } } , k , \Im } \left(\phi_{\Re}(y) \phi_{\Re}(y+n-k)\right.\right. \\
& \left.\left.\quad+\phi_{\Im}(y) \phi_{\Im}(y+n-k)\right)\right] \\
& +\sum_{k \in I_{n}}\left[\widetilde { c } _ { t _ { n _ { 1 } } , k , \Re } \left(\phi_{\Im}(x) \phi_{\Re}(y+n-k)\right.\right. \\
& \left.\left.\quad-\phi_{\Re}(y) \phi_{\Im}(y+n-k)\right)\right],
\end{aligned}
$$

where $\bar{v}_{n, f}\left(t_{n_{1}}\right):=u_{t_{n_{1}}, f}+\mathbf{i} v_{t_{n_{1}}, f}$ and

$$
\widetilde{c}_{t_{n_{1}}, k}:=\bar{\phi}\left(t_{n_{1}}\right) c_{k}=\widetilde{c}_{t_{n_{1}}, k, \Re}+\mathbf{i} \widetilde{\mathbf{c}}_{t_{n_{1}}, k, \Im} .
$$

As mention in section $\mathrm{V}-\mathrm{A}$, there exists at least one nonzero coefficient in $\left\{c_{k}: k \in I_{n}\right\}$ for every $n \in$ $\left\{1,2, \ldots, \mathcal{N}_{f}+s-1\right\}$. For any fixed $n \in\left\{1, \ldots, \mathcal{N}_{f}\right\}$, using $\Lambda_{\phi, 1}$ in Proposition 2.1 satisfying GHC, we have $P\left(\bar{\phi}\left(t_{n_{1}}\right) \neq 0\right)=1$, which together with (5.54) leads to that with probability 1 , there exists at least one nonzero coefficient in $\left\{\widetilde{c}_{t_{n_{1}}, k}: k \in I_{n}\right\}$. Then

$$
\begin{aligned}
& P\left(\Re\left(a_{n, f}\left(t_{n_{1}}, t_{n_{2}}\right)+\mathbf{i} b_{n, f}\left(t_{n_{1}}, t_{n_{2}}\right)\right) \neq 0\right) \\
\geq & P\left(\Re\left(a_{n, f}\left(t_{n_{1}}, t_{n_{2}}\right)+\mathbf{i} b_{n, f}\left(t_{n_{1}}, t_{n_{2}}\right)\right) \neq 0 \mid \mathfrak{E}_{n, 0}\right) \\
& \times P\left(\mathfrak{E}_{n, 0}\right) \\
= & P\left(\Re\left(a_{n, f}\left(t_{n_{1}}, t_{n_{2}}\right)+\mathbf{i} b_{n, f}\left(t_{n_{1}}, t_{n_{2}}\right)\right) \neq 0 \mid \mathfrak{E}_{n, 0}\right) \\
= & 1,
\end{aligned}
$$

where $P\left(\mathfrak{E}_{n, 0}\right)=1$, derived from section $\mathrm{V}$-A, is used in the first identity, and the second identity is derived from GHC (1.8). Therefore, $P\left(\Re\left(a_{n, f}\left(t_{n_{1}}, t_{n_{2}}\right)+\right.\right.$ $\left.\left.\mathbf{i} b_{n, f}\left(t_{n_{1}}, t_{n_{2}}\right)\right) \neq 0\right)=1$. Similarly, we can prove that $P\left(\Im\left(a_{n, f}\left(t_{n_{1}}, t_{n_{2}}\right)+\mathbf{i} b_{n, f}\left(t_{n_{1}}, t_{n_{2}}\right)\right) \neq 0\right)=1$. Then $P\left(\theta\left[a_{n, f}\left(t_{n_{1}}, t_{n_{2}}\right)+b_{n, f}\left(t_{n_{1}}, t_{n_{2}}\right) \mathbf{i}\right]=\frac{j \pi}{2}\right)=0$, where $j=0,1,2,3$. Applying the above result to $\tilde{f}:=$ $e^{\mathbf{i}\left(\frac{\pi}{2}-\alpha\right)} f \in V_{\mathrm{ca}}(\phi)$, the proof is concluded.

\section{Proof of Lemma 2.12}

Define three random events

$$
\begin{aligned}
\mathfrak{E}_{1}:=\{ & \left(A_{n, f}\left(t_{n_{1}}, t_{n_{2}}\right)+\mathbf{i} B_{n, f}\left(t_{n_{1}}, t_{n_{2}}\right)\right) \\
& \times\left(A_{n, f}\left(t_{n_{1}}, t_{n_{3}}\right)-\mathbf{i} B_{n, f}\left(t_{n_{1}}, t_{n_{3}}\right)\right) \\
& \neq\left(A_{n, f}\left(t_{n_{1}}, t_{n_{2}}\right)-\mathbf{i} B_{n, f}\left(t_{n_{1}}, t_{n_{2}}\right)\right) \\
& \left.\times\left(A_{n, f}\left(t_{n_{1}}, t_{n_{3}}\right)+\mathbf{i} B_{n, f}\left(t_{n_{1}}, t_{n_{3}}\right)\right)\right\},
\end{aligned}
$$

and

$$
\begin{aligned}
& \mathfrak{E}_{2}:=\left\{A_{n, f}\left(t_{n_{1}}, t_{n_{2}}\right)+\mathbf{i} B_{n, f}\left(t_{n_{1}}, t_{n_{2}}\right) \neq 0\right\}, \\
& \mathfrak{E}_{3}:=\left\{A_{n, f}\left(t_{n_{1}}, t_{n_{3}}\right)+\mathbf{i} B_{n, f}\left(t_{n_{1}}, t_{n_{3}}\right) \neq 0\right\} .
\end{aligned}
$$

Next we prove that $P\left(\mathfrak{E}_{1}\right)=1$. By Lemma 2.10, $P\left(\mathfrak{E}_{2}\right)=$ $P\left(\mathfrak{E}_{3}\right)=1$. Direct computation gives that

$$
\begin{aligned}
1 & \geq P\left(\mathfrak{E}_{1}\right) \\
& \geq P\left(\mathfrak{E}_{1} \cap \mathfrak{E}_{2}\right) \\
& =P\left(\mathfrak{E}_{1} \mid \mathfrak{E}_{2}\right) P\left(\mathfrak{E}_{2}\right) \\
& =P\left(\mathfrak{E}_{1} \mid \mathfrak{E}_{2}\right) .
\end{aligned}
$$


By (5.55) and (5.56), we have

$$
\begin{aligned}
& \mathfrak{E}_{1} \mid \mathfrak{E}_{2} \\
& =\left\{A_{n, f}\left(t_{n_{1}}, t_{n_{3}}\right)-\mathbf{i} B_{n, f}\left(t_{n_{1}}, t_{n_{3}}\right)\right. \\
& \left.-b\left(t_{n_{1}}, t_{n_{2}}\right)\left(A_{n, f}\left(t_{n_{1}}, t_{n_{3}}\right)+\mathbf{i} B_{n, f}\left(t_{n_{1}}, t_{n_{3}}\right)\right) \neq 0 \mid \mathfrak{E}_{2}\right\},
\end{aligned}
$$

where

$$
b\left(t_{n_{1}}, t_{n_{2}}\right)=\frac{A_{n, f}\left(t_{n_{1}}, t_{n_{2}}\right)-\mathbf{i} B_{n, f}\left(t_{n_{1}}, t_{n_{2}}\right)}{A_{n, f}\left(t_{n_{1}}, t_{n_{2}}\right)+\mathbf{i} B_{n, f}\left(t_{n_{1}}, t_{n_{2}}\right)} .
$$

Applying Lemma 2.11 to $A_{n, f}\left(t_{n_{1}}, t_{n_{3}}\right)+\mathbf{i} B_{n, f}\left(t_{n_{1}}, t_{n_{3}}\right)$, it is easy to prove that $P\left(\mathfrak{E}_{1} \mid \mathfrak{E}_{2}\right)=1$ which together with (5.57) leads to $P\left(\mathfrak{E}_{1}\right)=1$. Now the rest of proof can be easily concluded.

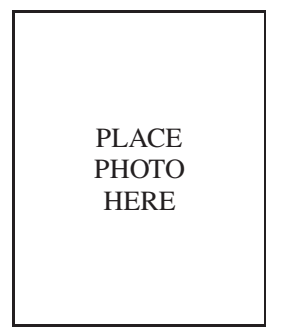

Youfa Li Biography text here.

Wenchang Sun Biography text here.

PLACE

PHOTO

HERE

\section{REFERENCES}

[1] R. W. Gerchberg and W. O. Saxton, A practical algorithm for the determination of phase from image and diffraction plane pictures, Optik, 35, 237-246, 1972.

[2] J. R. Fienup, Phase retrieval algorithms: A comparison, Applied Optics, 21(15), 2758-2769, 1982.

[3] J. R. Fienup, Reconstruction of an object from the modulus of its Fourier transform, Optics Letters, 3(1), 27-29, 1978.

[4] J. R. Fienup, Phase retrieval algorithms: A personal tour, Applied Optics, 52(1), 45-56, 2013.

[5] E. J. Candès, T. Strohmer and V. Voroninski, PhaseLift: Exact and stable signal recovery from magnitude measurements via convex programming, Communications on Pure and Applied Mathematics, 66(8), 1241-1274, 2013.

[6] B. A. Shenoy and C. S. Seelamantula, Exact phase retrieval for a class of 2-D parametric signals, IEEE Transactions on Signal Processing, 63(1), 90-103, 2015.

[7] Y. Shechtman, Y. C. Eldar, O. Cohen, H. N. Chapman, J. Miao and M. Segev, Phase retrieval with application to optical imaging, IEEE Signal Processing Magazine, 32(3), 87-109, 2015.

[8] J. Miao, T. Ishikawa, I.K. Robinson and M.M. Murnane, Beyond crystallography: Diffractive imaging using coherent $\mathrm{x}$-ray light sources, Science, 348(6234), 530-535, 2015.

[9] E. J. Candès, Y. C. Eldar, T. Strohmer and V. Voroninski, Phase retrieval via matrix completion, SIAM Journal on Imaging Sciences, 6(1), 199-225, 2013.

[10] T. Heinosaarri, L. Mazzarella and M. M.Wolf, Quantum tomography under prior information, Communications in Mathematical Physics, 318, 355-374, 2013.

[11] R. Balan, P.G. Casazza and D. Edidin, On signal reconstruction without noisy phase, Applied and Computational Harmonic Analysis, 20, 345-356, 2006.

[12] L. Li, C. Cheng, D. Han, Q. Sun and G. Shi, Phase retrieval from multiple-window short-time Fourier measurements, IEEE Signal Processing Letters, 24(4), 372-376, 2017.

[13] K. Huang, Y. C. Eldar and N. D. Sidiropoulos, Phase retrieval from 1D Fourier measurements: convexity, uniqueness, and algorithms, IEEE Transactions on Signal Processing, 64(23), 6105-6117, 2016.

[14] Y. Chen, C. Cheng and Q. Sun, Phase retrieval of complex and vector-valued functions, arXiv preprint arXiv: 1909.02078v1.

[15] R. Alaifari, I. Daubechies, P. Grohs and G. Thakur, Reconstructing real-valued functions from unsigned coeffcients with respect to wavelet and other frames, Journal of Fourier Analysis and Applications, 23, 1480-1494, 2017.

[16] R. Alaifari, I. Daubechies, P. Grohs and R. Yin, Stable phase retrieval in infinite dimensions, Foundations of Computational Mathematics, 19, 869-900, 2019.

[17] J. Cahill, P. G. Casazza and I. Daubechies, Phase retrieval in infinite-dimensional Hilbert spaces, Trans. Amer. Math. Soc. Ser. B, 3, 63-76, 2016.

[18] N. Shlezinger, R. Dabora and Y. C. Eldar, Measurement matrix design for phase retrieval based on mutual information, IEEE Transactions on Signal Processing, 66(2), 324-339, 2018.

[19] B. A. Shenoy, S. Mulleti and C. S. Seelamantula, Exact phase retrieval in principal shift-invariant spaces, IEEE Transactions on Signal Processing, 64(2), 406-416, 2016.

[20] A. Aldroubi and K. Gröchenig, Nonuniform sampling and reconstruction in shift-invariant spaces, SIAM Rev., 43, 585-620, 2001.

[21] J. Shi, X. Liu, F. G Yan and W. Song, Error analysis of reconstruction from linear canonical transform based sampling, IEEE Transactions on Signal Processing, 66(7), 1748-1760, 2018.

[22] A. Bhandari and A. I. Zayed, Shift-Invariant and sampling spaces associated with the fractional Fourier transform domain, IEEE Transactions on Signal Processing, 60(4), 1627-1637, 2012.

[23] W. Sun, Phaseless sampling and linear reconstruction of functions in spline spaces, arXiv preprint, arXiv:1709.04779.

[24] W. Sun, Local and global phaseless sampling in real spline spaces, arXiv preprint, arXiv:1705.00836. 
[25] G. Thakur, Reconstruction of bandlimited functions from unsigned samples, Journal of Fourier Analysis and Applications, 17(4), 720732, 2011.

[26] Y. Chen, C. Cheng, Q. Sun and H. Wang, Phase retrieval of realvalued signals in a shift-invariant space, Applied and Computational Harmonic Analysis, 49, 56-73, 2020.

[27] C. Cheng, J. Jiang and Q. Sun, Phaseless sampling and reconstruction of real-valued signals in shift-invariant spaces, Journal of Fourier Analysis and Applications, 25, 1361-1394, 2019.

[28] P. Jaming, K. Kellay and R. Perez Iii, Phase retrieval for wide-band signals, arXiv preprint arXiv:1905.04095.

[29] C.K. Lai, F. Littmann and E. Weber, Conjugate phase retrieval in Paley-Wiener space, arXiv preprint arXiv: 1910.12975.

[30] J. Selva, Interpolation of bounded bandlimited signals and applications, IEEE Transactions on Signal Processing, 54(11), 4244-4260, 2006.

[31] B. Han, Framelets and wavelets: Algorithms, analysis, and applications, Applied and Numerical Harmonic Analysis, Birkhäuser/Springer, Cham, 2017. xxxiii +724 pp.

[32] R. Marks, J. Walkup and M. Hagler, Sampling theorems for linear shift-variant systems, IEEE Transactions on Circuits and Systems, 25(4), 228-233, 1978

[33] G. Meinsma and L. Mirkin, $L^{2}$ Sampled signal reconstruction with causality constraints - Part I: Setup and solutions, IEEE Transactions on Signal Processing, 60(5), 2260-2272, 2012.

[34] A. Bhandari and A. I. Zayed, Shift-invariant and sampling spaces associated with the special affine Fourier transform, Applied and Computational Harmonic Analysis, 47(1), 30-52, 2019.

[35] D. Han, Frame representations and Parseval duals with applications to Gabor frames, Transactions of the American Mathematical Society, 360(6), 3307-3326, 2008.

[36] D. Han and D. Larson, Frames, bases and group representations, Memoirs of the American Mathematical Society, 697, 2000.

[37] C. K. Chui, P. W. Swih and J. D. Ward, Best $L_{2}$ local approximation, Journal of Approximation Theory, 22, 254-261, 1978.

[38] C. B Dunham, Families satisfying the Haar condition, Journal of Approximation Theory, 12, 291-298, 1974.

[39] H. Van de Vel, The Haar condition and multiplicity of zeros, Numerische Mathematik, 39, 139-153, 1982.

[40] E. J. Candès, P. R. Charlton and H. Helgason, Detecting highly oscillatory signals by chirplet path pursuit, Applied and Computational Harmonic Analysis, 24(1), 14-40, 2008.

[41] J. Simmons, Echolocation in bats: Signal processing of echoes for target range, Science, 171 (974), 925-928, 1971.

[42] J.E. Reynolds III and S.A. Rommel, Biology of Marine Mammals, Smithsonian Institution Press, Washington, DC, 1999.

[43] X. Gu, S. Akturk and R. Trebino, Spatial chirp in ultrafast optics, Optics Communications, 242(4), 599-604, 2004.

[44] S. Akturk, X. Gu, P. Bowlan and R. Trebino, Spatio-temporal couplings in ultrashort laser pulses, Journal of Optics, 12, 093001, 2010.

[45] J. Garnett, Bounded analytic functions, Graduate Texts in Mathematics, 236, Springer, 2007.

[46] T. N. T. Goodman and C. A. Micchelli, On refinement equations determined by Pólya frequency sequences, SIAM Journal on Mathematical Analysis, 23, 766-784, 1992.

[47] T. N. T. Goodman and Q. Sun, Total positivity and refinable functions with general dilation, Applied and Computational Harmonic Analysis, 16, 69-89, 2004.

[48] S. Yang and Y. Li, Two-direction refinable functions and twodirection wavelets with high approximation order and regularity, Science in China Series A: Mathematics, 50, 1687-1704, 2007. 